\title{
1 Lipid bilayers: phase behavior and 2 nanomechanics
}

3

4

9 Biomedical Research Networking Center on Bioengineering, Biomaterials and Nanomedicine 10

LORENA REDONDO-MORATA

Center for Infection and Immunity of Lille, INSERM U1019, CNRS UMR 8204, F-59000 Lille, France.

PATRicia Losada-PÉRez

Experimental Soft Matter and Thermal Physics (EST) group, Department of Physics, Université Libre de Bruxelles, 1050 Brussels, Belgium

\section{MARINA INÉS GIANNOTTI} (CIBER-BBN), Spain.

Institut de Bioenginyeria de Catalunya (IBEC), The Barcelona Institute of Science and Technology (BIST), 08028 Barcelona, Spain.

Departament de Ciència de Materials i Química Física, Universitat de Barcelona, 08028 Barcelona, Spain.

migiannotti@ibecbarcelona.eu

\section{Keywords:}

Lipid phase behavior / phase transition / phase coexistence / nanomechanics / thermodynamics / Atomic Force Microscopy (AFM)/ Quartz crystal microbalance with dissipation monitoring (QCM-D) 
Contents

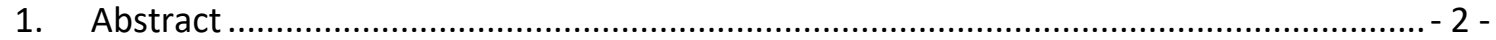

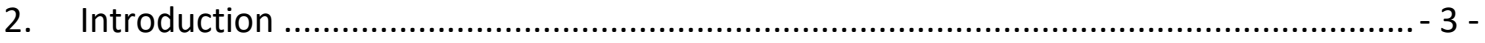

3. Experimental approaches to study membrane mechanics ............................................... - 5 -

3.1. Model systems: supported vesicles layers (SVLs) lipid bilayers (SLBs) .......................... - 5 -

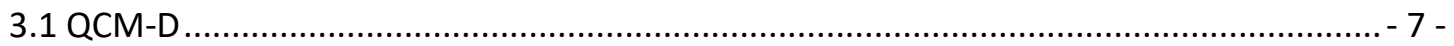

3.2. Atomic Force Microscopy (AFM)-based methodology .............................................. 11 -

4. Phase behavior and nanomechanics. From one component membranes to higher complexity -18 -

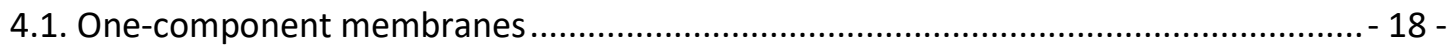

4.1.1. The gel and the fluid phase ….......................................................................... 18 -

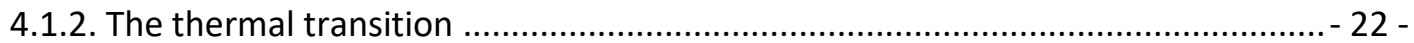

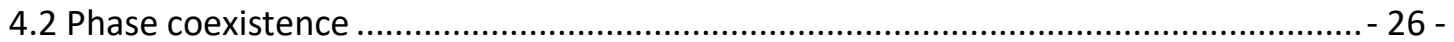

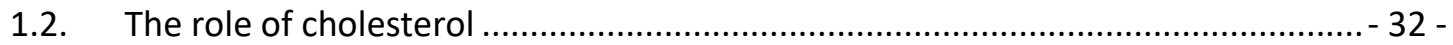

5. Connection between nanoscale measurements and thermodynamic descriptors of membranes $-37-$

6. Conclusions and future perspectives ............................................................................ 38

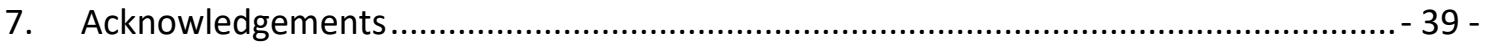

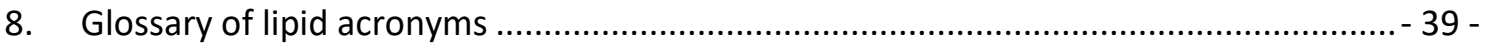

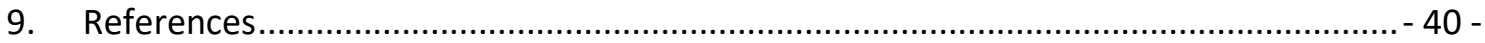

\section{Abstract}

Lipid membranes are involved in many physiological processes like recognition, signaling, fusion or remodeling of the cell membrane or some of its internal compartments. Within the cell, they are the ultimate barrier, while maintaining the fluidity or flexibility required for a myriad of processes, including membrane protein assembly. The physical properties of in vitro model membranes as model cell membranes have been extensively studied with a variety of techniques, from classical thermodynamics to advanced modern microscopies. Here we review the nanomechanics of solid-supported lipid membranes with a focus in their phase behavior. Relevant information obtained by quartz crystal microbalance with dissipation monitoring (QCM-D) and atomic force microscopy (AFM) as complementary techniques in the nano/mesoscale interface is presented. Membrane morphological and mechanical characterization will be discussed in the framework of its phase behavior, phase transitions and coexistence, in simple and complex models, and upon the presence of cholesterol. 


\section{Introduction}

Cells can be thermodynamically defined as open systems in constant exchange of mass, energy and information with the environment. The membrane is the ultimate boundary for the cell, confining it from the medium and some of its internal compartments. The cell membrane is a fundamental structure of the cell, providing a support matrix for proteins and carbohydrates. As complex systems, cell membrane performance is the result of lipids and proteins working together, with main functions like being barriers, mediating the exchange of molecules and information, promoting signaling and adhesion, and being metabolically self-renewing structures.

Cell membranes are materials with unique physical properties allowing cells to rapidly change shape, squeeze, stretch, pinch off smaller units, fuse, and reseal. This is the result of being viscous sheets with both fluid and elastic properties. Cell membranes are supramolecular structures, where the lipids and proteins interact through non-covalent bonding. Membranes are curved surfaces, vesicular in nature, whose curvature is deeply influenced by the lipid packing. The lipid bilayer, made of millions of building blocks held together by weak interactions, is in part responsible for the physical, dynamic and mechanical properties of the cell membrane. In the membrane, lipids are mainly organized in lamellar phases, where two leaflets of lipids are self-assembled exposing the polar headgroups to the aqueous interface and keeping the fattyacyl chains aligned opposed and parallel to one another to form the hydrophobic core.

The enterprising scientist Alec Bangham was the discoverer in 1958 of the 'multilamellar smectic mesophases' or, as himself referred to less seriously, the 'bangasomes' (Bangham et al., 1958) which were eventually named 'liposomes' to define microscopic lipid vesicles (Sessa \& Weissmann, 1968). When the Babraham Institute (Cambridge, UK) acquired its first electron microscope in 1961, Bangham had the privilege to firstly observe the dispersions of phospholipids in water solutions of negative stains (Bangham \& Horne, 1964). In the following years, Bangham and his collaborators performed the key experiments to demonstrate that lipid bilayers maintain concentration gradients of ions such as potassium and sodium. Indeed, David D. Deamer wrote in his memoir "It was the membrane equivalent of finding the double helix structure of DNA, another Cambridge discovery in the life sciences"(Deamer, 2010). This was reflected in the well-known lecture by Bangham in the University of Bristol in 1975, entitled "Membranes came first", where he proposed that something similar to liposomes had been available to house the first forms of life. Indeed, all living organisms have a membrane. 
Later in the early 1970s, Singer and Nicolson proposed the fluid-mosaic-model, depicturing the cell membranes as two-dimensional liquids where all lipid and protein molecules diffuse easily (Singer \& Nicolson, 1972). Concerning the composition, besides all the proteins and carbohydrates, lipids are the main components of the cell membrane in terms of molar fraction. Lipids are a broad family which covers many different chemical structure sphingolipids (ceramides, sphingomyelin, gangliosides, sphingosines), sterols (cholesterol and vitamins), and phospholipids, each of them contributing to different and crucial physicochemical properties.

Gradual progress was made in the knowledge of the complexity of the biological membranes. The most famous hypothesis in the field is the membrane raft proposed in 1997 by K. Simons and E. Ikonen (Simons \& Ikonen, 1997). The idea was based on the fact that portions of membrane were found to be detergent resistant, that may give rise to a virtual compartmentalization of the cell. This hypothesis generated a lot of literature and became a recursive concept in the field. Nowadays it is well known that there is a variety of nanostructures in the membrane of heterogeneous sizes and functions, and the methods that allow us to observe these nanodomains in vivo are only beginning to emerge (Goñi, 2019b; Pinkwart et al., 2019). Nanodomains can be considered putative heterogeneous structures within the membrane which are due partly to phase separation.

The mechanical role of the lipid membrane in force-triggered and force-sensing mechanisms in cells is of significance and adds to the better-established role of the mechanosensitive proteins (Vogel, 2006)(Kechagia et al., 2019). Membrane conformational changes such as bending, vesiculation or tubulation are involved in cellular processes including adhesion, signaling, endocytosis or membrane resealing (van Meer \& de Kroon, 2011)(van Meer et al., 2008)(Hassinger et al., 2017). For instance, in endocytosis, the endocytic system needs to generate enough force to form an endocytic vesicle by bending the membrane. These mechanisms generally require the membrane separation from the cytoskeleton as well as strong bending, for which the membrane chemical composition and physicochemical properties, often highly localized and dynamic, are key players (Sheetz, 2001)(van Meer et al., 2008). It is now clear that the lipid packing and composition are direct modulators of the membrane curvature and elasticity, at different scales, even locally at the nanometer scale (Yeagle, 1989)(Vereb et al., 2003). Understanding the lipid bilayers structural and mechanical properties and the involvement and role of each individual component turns out to be essential in order to identify their contribution to the overall membrane traits. Structural and physical properties of lipid bilayers include shape and local distribution of components (phases and domains), and related mechanical stability in response to compression, bending or stretching, their ability to fuse, etc. 
122 We review the nanomechanics of solid-supported lipid membranes and how these relate to lipid

123 membrane phase behavior. To this end, we chose two complementary techniques with

124 nano/mesoscale sensitivity to mechanical and viscoelastic properties, namely quartz crystal

125 microbalance with dissipation monitoring (QCM-D) and atomic force microscopy (AFM). QCM-D

126 is a label-free surface-sensitive technique, whose working principle is based on the inverse

127 piezoelectric effect. Real-time simultaneous measurements of resonant frequency and energy

128 dissipation make QCM-D very suitable for gravimetric and viscoelastic characterization of solid-

129 supported nanoscale sized films. AFM is a technique that allows the observation of surfaces

130 under controlled liquid environment, to resolve topographical features with nanoscale

131 resolution and to measure and apply forces in the $\mathrm{pN}-\mathrm{nN}$ range. Therefore, AFM is appropriate

132 for imaging the topography of solid-supported lipid membranes and probing the physical and

133 mechanical properties at the nanoscale by means of force spectroscopy, providing high spatial

134 and force resolution. QCM-D is complementary to AFM, useful for probing changes in

135 viscoelastic properties during solid-supported film formation. The complementarity of AFM and

136 QCM-D manifests at different levels: i) AFM measurements are performed at a local level, while

137 QCM-D provides a global characterization of the solid-supported films; ii) both techniques

138 enable dynamic mechanical analysis by applying a small oscillatory stress, however, the AFM tip

139 scans and deforms supported layers from the top, whereas QCM-D measures the sensor

140 oscillation from the bottom. The combination of AFM and QCM-D for supported lipid

141 membranes has been employed in previous works (see, for instance (R. Richter et al., 2003,

142 2003; Van Lehn et al., 2014). In the following sections we briefly describe the membrane models

143 and discuss the working principles behind both approaches, highlighting the quantitative and

144 qualitative mechanical information that can be extracted using each technique. Specific

145 examples on the usefulness to monitor phase transitions on one-component to more complex

146 bilayers, and resolve domain coexistence are also provided, including the key role of cholesterol.

\section{Experimental approaches to study membrane mechanics}

\subsection{Model systems: supported vesicles layers (SVLS) lipid bilayers (SLBs)}

Due to the extreme complexity of biological membranes, cell membrane mimetics are excellent approaches to study membrane properties and biological processes at the cellular and subcellular level. A great deal of what we know about these processes comes from modeling lipid bilayers in vitro. Various membrane systems are established as biomimetic structures. They include vesicles: freely-suspended and supported liposomes, giant unilamellar vesicles (GUVs); 
lipid membranes (tBLM), polymer-cushioned membranes, protein-tethered bilayer lipid membranes (ptBLM) and hybrid bilayers (Sebaaly et al., 2019)(Dimova, 2019)(Doktorova et al., 2018)(Siontorou et al., 2017)(Rascol et al., 2016). Among these, the giant unilamellar vesicles (GUVs) have been extensively used since they offer a perfect stage to study the mechanical, thermodynamic, electrical, and rheological properties of the overall GUV and lipid bilayer as a function of membrane composition, surrounding media and temperature (Dimova, 2019)(Evan Evans et al., 2003)(Kahya et al., 2004). Being heterogeneous and dynamic at the nanoscale, nanotechnology-based techniques can further explore biomembranes locally with resolution at the nanometric level. For many of these techniques, like surface analytical techniques, solidsupported models are the most adequate, including supported lipid monolayers, supported vesicles layers (SVLS) or supported lipid bilayers (SLBs).

SVLs are the precursor solid-supported systems to planar SLBs formed by vesicle fusion and rupture. They result from spontaneous adsorption of small vesicles of diameter $d \leq 200 \mathrm{~nm}$ onto solid surfaces. The geometry of SVLs embodies membrane curvature, tension and osmotic stress within the supported layer. This makes SVLs useful biomimetic platforms to probe membrane deformation using surface-sensitive techniques and testing ground for adhesion, budding and lipid membrane exchange and fusion (Lipowsky \& Seifert, 1991; Hurley et al., 2010; Tabaei et al., 2016; Steinkühler et al., 2019). SVLs are generally formed by small soft adsorbates which are mimics to small endosomes or exosomes. These are optically inaccessible systems and the experimental investigation of their deformation is not straightforward. Adsorbed vesicles onto inorganic surfaces also serve as model systems relevant to biocompatibility studies. Vesicle adsorption is very often accompanied by vesicle deformation upon contact with the solid support. The extent of vesicle deformation depends on several factors such as vesicle size, mechanical properties of the vesicles, adhesion strength of the surface, osmotic pressure difference over the vesicle, etc. The fate of vesicles upon adsorption on a solid support depends both on vesicle-vesicle and vesicle surface interactions. In the absence of osmotic stress and at a concentration where vesicle surface coverage enhances fusion probability, the rupture of vesicles will depend strongly on the adhesion strength. Specifically on how this imbalances the energetic competition between adhesion energy and an elastic stretching of the membrane (Lipowsky \& Seifert, 1991). If the adhesion strength $W$ is large enough and exceeds the lysis tension of the membrane, the vesicles will rupture. SVLs are typically formed on surfaces whose adhesion strength is not sufficient to induce vesicle rupture, such as $\mathrm{Au}^{\mathrm{TiO}} \mathrm{Ti}_{2}, \mathrm{Pt}$ (Tero, 2012). 
190

191

192

193

194

195

196

197

198

199

200

201

202

203

204

205

206

207

208

209

210

211

212

213

214

215

216

217

218

219

221

222

mechanical properties, growth of lipid domains, as well as interactions between the lipid membrane and proteins, peptides and drugs, cell signaling, etc. They also offer an excellent environment for inserting membrane proteins. There are many different methods that can be used to obtain SLBs, like the spin-coating and hydration (Mennicke \& Salditt, 2002), microcontact printing (Strulson \& Maurer, 2011), solvent-exchange deposition (Tabaei et al., 2016; Hohner et al., 2010), and the most widely used methods like the LangmuirBlodgett/Schäfer deposition to prepare mono and bilayers (Kurniawan et al., 2018), and the liposome fusion and rupture method for bilayers (Hardy et al., 2013)(Mingeot-Leclercq et al., 2008). The liposome rupture method remains the most popular and simple one, based on depositing small unilamellar vesicles (SUVs) from a suspension onto a flat substrate where the adhesion strength is high, generally mica or silicon oxide, but can also be formed on bare or gold-coated glass, following the needs of the analysis technique (Mingeot-Leclercq et al., 2008; Ralf P Richter \& Brisson, 2005)(Choi et al., 2016; B. Gumí-Audenis et al., 2018; Seeger et al., 2010; Winkler et al., 2020). Once in contact with the substrate the SUVs start fusing between them, deforming, flattening, and finally rupturing to form a continuous film. It is important to have in mind that the final SLB structure is affected by variables like the lipid vesicles composition, concentration, and size, the physicochemical environment $\mathrm{pH}$, temperature, and ionic strength, as well as the surface roughness and charge density (Reimhult et al., 2003).

\subsection{QCM-D}

Quartz crystal microbalance with dissipation monitoring (QCM-D) is an acoustic-based surfacesensitive technique that enables label-free, real-time simultaneous measurements of wet-mass and viscoelastic properties of solid-supported nano/meso-scaled adlayers.

The QCM-D sensor consists of an AT-cut quartz crystal being sandwiched between two electrodes. When an AC voltage is applied across the electrodes with a frequency close to the resonant frequency of the quartz crystal, a mechanical deformation is induced, resulting in a standing shear wave. The surfaces of the electrodes coincide with the antinodes of the standing shear wave with wavelength $\lambda=2 d / n$, with $d$ the quartz thickness and $n$ the (odd) overtone number. The resonance frequency at each overtone is $f_{n}=n v / 2 d$, with $v$ the speed of sound in quartz. The shear waves propagate as evanescent waves decaying across the boundary between the crystal and the fluid environment (air or liquid) with a penetration depth $\delta=\sqrt{\eta_{L} / \pi f_{n} \rho_{L}}$, which depends on the overtone frequency $f_{n}$ and on the viscosity $\eta_{L}$ and density $\rho_{L}$ of the fluid in contact with the sensor surface (Reviakine et al., 2011). The penetration depth of a $5 \mathrm{MHz}$ shear wave in water is $\delta \sim 250 \mathrm{~nm}$, rendering QCM-D surface-specific. $D$ is defined as the ratio 
between the dissipated energy during one vibration period and the total energy of the crystal at that instant $D=E_{\text {lost }} / 2 \pi E_{\text {stored }}$. In the so-called 'ring-down method' measurements, energy dissipation $D$ can be calculated when the AC voltage driving the quartz crystal oscillation is turned off. The working principle of QCM-D is reflected in Figure 1, upon the presence of a soft, viscoelastic layer such as a layer of lipid SVLs. The amplitude of the crystal oscillation decays exponentially and much faster when in contact with a viscoelastic layer, characterized by a large energy dissipation response.
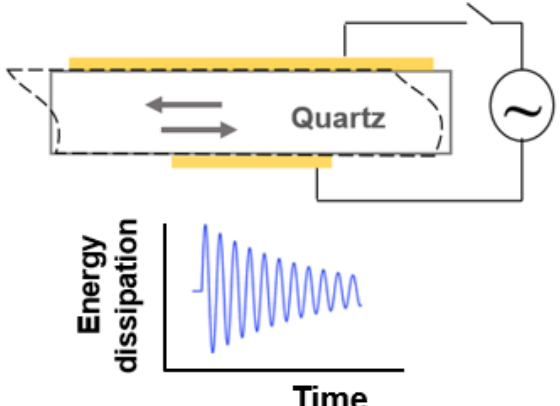
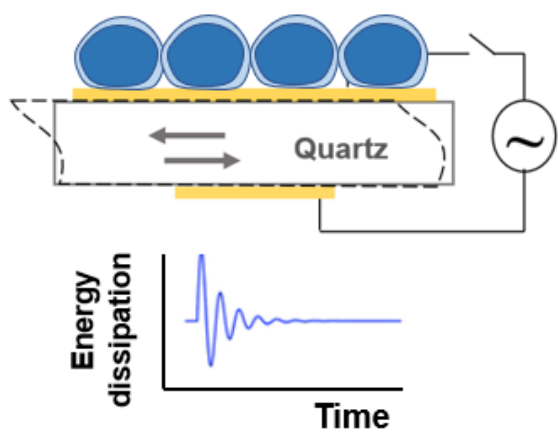

Figure 1. Schematic view of a QCM-D sensor excited by an AC voltage. Left panel: Bare Au-coated sensor in liquid environment, the dampening of the oscillations takes place slowly. Right panel: The presence of an adsorbed lipid vesicle layer (SVL) induces a faster damping of the oscillations and thus a larger energy dissipation.

Commercial QCM-D instruments allow for monitoring changes in frequency and dissipation for several overtones $(n=3,5,7, \ldots, 13), \Delta f_{n}$ and $\Delta D_{n}$, upon the presence of a nano/mesoscale layer. The spectroscopic data at multiple frequencies with varying detection range enables assessing the spatial homogeneity and rigidity of the adsorbed layer. For homogeneous thin and rigid films, $\Delta D_{\mathrm{n}} \sim 0, \Delta f_{\mathrm{n}}$ overtones overlap and a simple relationship between mass adsorbed and change in frequency holds, the so-called Sauerbrey relation: $\Delta m=-C \Delta f / n$, with $C$ being the Sauerbrey constant which for a $5 \mathrm{MHz}$ quartz crystal reads $C=d \rho_{\text {quartz }} / f_{0}=17.7 \mathrm{ng} / \mathrm{cm}^{2}$ (Sauerbrey, 1959). For thicker and softer films $\Delta D_{\mathrm{n}}>0$, overtones do not overlap and the ensemble quartz-film-fluid can be represented by simple models combining elastic components and viscous dashpots. These models enable to extract effective viscoelastic parameters of the adsorbed film such as shear viscosity, thickness and shear storage modulus (Cho et al., 2007; Voinova et al., 1999).

The capability of monitoring the viscoelastic properties during film formation is particularly useful in the case of solid-SLB formation. QCM-D has been a valuable tool in deciphering the kinetic pathways of SLB formation from precursor vesicles and how those depend on relevant 
conditions such as solid surface adhesion strength, medium ionic strength, vesicle mechanics, etc. (Cho et al., 2010; Jing et al., 2013; C. A. Keller \& Kasemo, 1998; Pramanik et al., 2016; R P Richter, 2006).

Figure 2 shows a typical QCM-D experiment where real-time monitoring of the adsorption of zwitterionic DOPC vesicles onto $\mathrm{SiO}_{2}$ and oxidized Au was carried out at $37^{\circ} \mathrm{C}$. The vesicles are dispersed in TRIS-buffer (10 mM TRIS, $100 \mathrm{mM} \mathrm{NaCl}, \mathrm{pH}$ ) at a concentration of $0.1 \mathrm{mg} / \mathrm{mL}$ and their hydrodynamic diameter $d=79 \pm 20 \mathrm{~nm}$. As initially discussed by Keller and Kasemo (C. A. Keller \& Kasemo, 1998), the adsorption kinetics is surface-specific. The mechanistic picture of $\Delta f$ and $\Delta D$ changes is governed by a delicate balance between the adhesive contribution to the free energy from lipid-surface interactions and the opposing effect of bending and stretching the membrane (Lipowsky \& Seifert, 1991). DOPC vesicles adsorbing on $\mathrm{SiO}_{2}$ follow a two-step adsorption process consisting of i) adsorption of a critical number of vesicles and ii) fusion, rupture and formation of an SLB. This is reflected in the $\Delta f_{\mathrm{n}}$ and $\Delta D_{\mathrm{n}}$ signals depicted in the upper panels of Figure 2. After a $\sim 10 \mathrm{~min}$ baseline in buffer $\left(\Delta f_{\mathrm{n}}\right.$ and $\left.\Delta D_{\mathrm{n}}=0\right)$, DOPC vesicles added at a very small flow rate adsorb onto the $\mathrm{SiO}_{2}$-coated quartz sensor $\left(\Delta f_{\mathrm{n}}\right.$ decreases and $\Delta D_{\mathrm{n}}$ increases) until a maximum number (minimum in $\Delta f_{\mathrm{n}}$ and maximum in $\Delta D_{\mathrm{n}}$ ). Adsorbed vesicles then fuse and rupture as a consequence of the large adhesion strength of $\mathrm{SiO}_{2}$, thus releasing the entrapped aqueous buffer. As a consequence, $\Delta f_{n}$ increases (mass loss) to a constant plateau of $\Delta f_{\mathrm{n}} \sim-25 \mathrm{~Hz}$ and the $\Delta D_{\mathrm{n}}$ signal decreases back to a very small value $\left(\Delta D_{\mathrm{n}}<0.5 \cdot 10^{-6}\right)$. The final $\Delta f_{\mathrm{n}}$ and $\Delta D_{\mathrm{n}}$ values and the fact that overtones signals overlap is consistent with the formation of a homogeneous rigid and thin DOPC SLB. The pathway of SLB formation is not unique and depends strongly on the ionic conditions (head group charges of the constituent lipids, buffer ionic strength presence of divalent cations, $\mathrm{pH}$ ). Vesicles containing positively charged lipids like DOTAP SUVs rupture individually on $\mathrm{SiO}_{2}$ as a consequence of stronger vesicle-support electrostatic interactions (R. Richter et al., 2003).

When DOPC vesicles adsorb onto an oxidized Au surface, a monotonic $\Delta f_{\mathrm{n}}$ decrease and $\Delta D_{\mathrm{n}}$ increase can be observed reaching constant non-zero plateau values with non-overlapping overtones. Such time-dependent responses provide evidence that oxidized Au facilitates nonruptured vesicle adsorption towards the formation of acoustically non-rigid vesicle layers with saturated coverage. As pointed out by Lind and Cárdenas (Lind \& Cárdenas, 2016), it is worth mentioning that local vesicle rupture events and formation of small bilayer patches cannot be ruled out. As a matter of fact, QCM-D is very sensitive to hydrodynamic (wet) mass and the local, partial formation of SLBs might be masked by the adsorption of vesicles on top or in between the bilayer patches. In this case, complementary QCM-D and AFM measurements are 

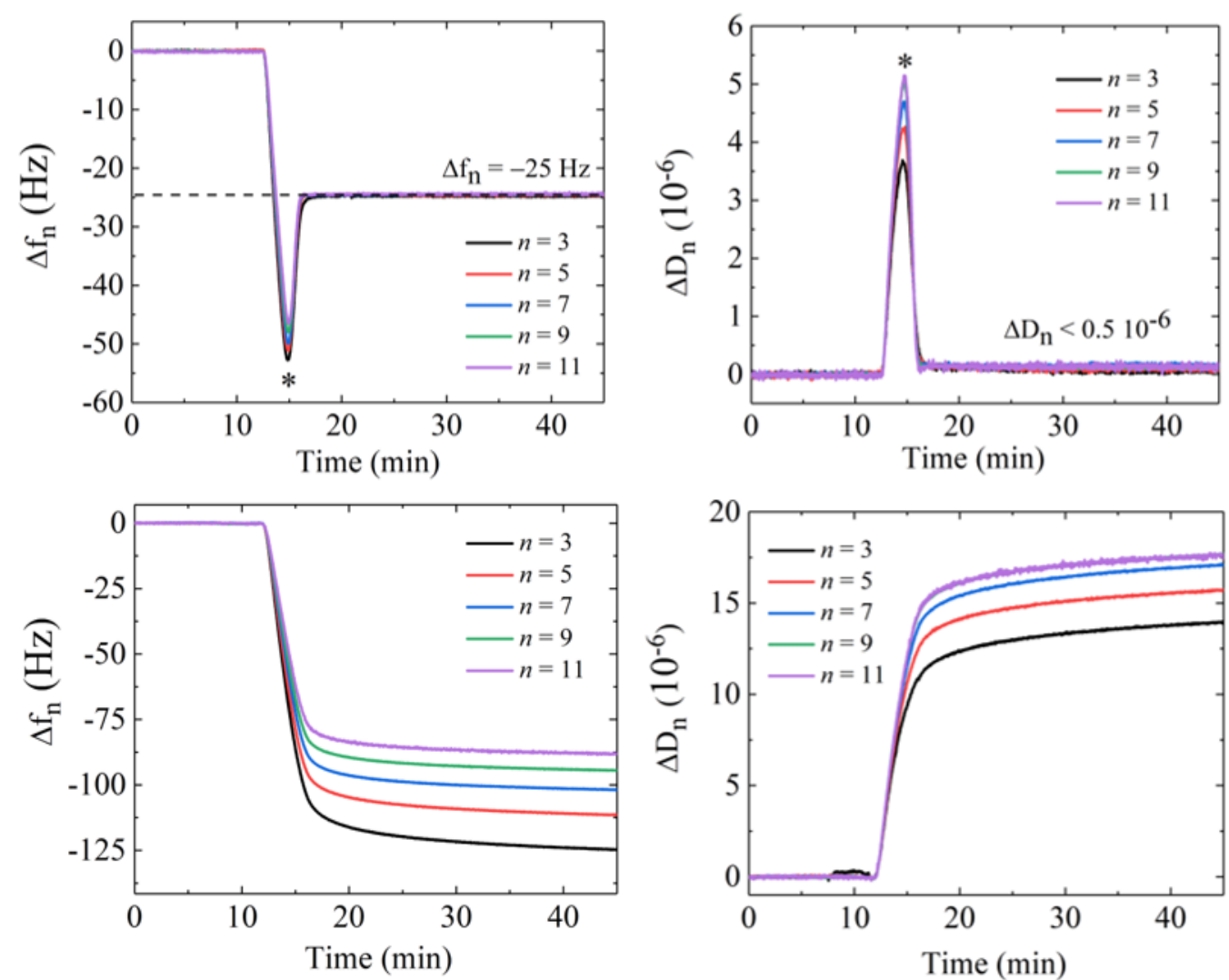

particularly useful to obtain a complete picture of the heterogeneous layers SLBs with coadsorbed vesicles formed. For example, the complementarity of AFM and QCM-D has proven successful in experimentally confirming defect (protrusion)-driven nanoparticle interactions with SLBs (Van Lehn et al., 2014).

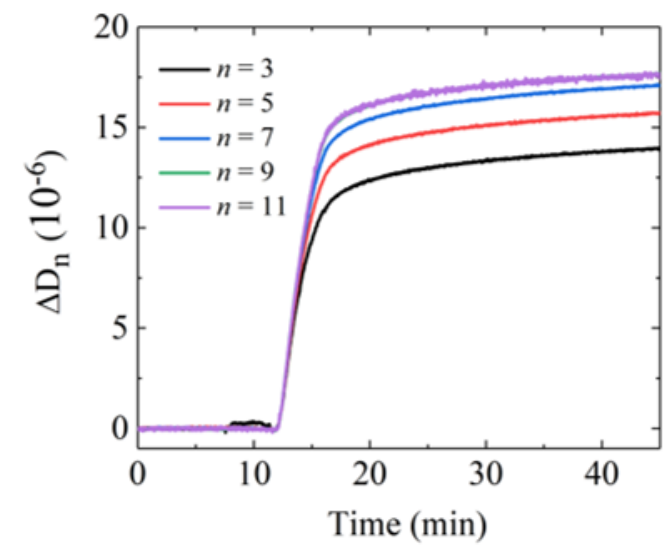

289 Figure 2. Time dependence of $\Delta f_{\mathrm{n}}$ and $\Delta D_{\mathrm{n}}$ during a QCM-D experiment of DOPC vesicle adsorption 290 at $37^{\circ} \mathrm{C}$ onto $\mathrm{SiO}_{2}$ (upper panels) and $\mathrm{Au}$ (lower panels).

291 Apart from kinetic information, QCM-D can be also useful to estimate the extent of vesicle deformation upon adsorption following a model-free approach introduced by Tellechea et al. (Tellechea et al., 2009). This method consists in plotting $-\Delta D / \Delta f$ ratio vs $-\Delta f$ for all overtones during initial adsorption, which shows a linear decrease over a large range of frequency shifts. Extrapolation of this linear decrease to a frequency-independent intercept with the $-\Delta f$ axis (where overtones intersect) provides a value of the thickness of the adsorbed vesicle layer $h$ or Sauerbrey thickness: $h=-\Delta f C / \rho$, where $C=17.7 \mathrm{ng} / \mathrm{cm}^{2} \mathrm{~Hz}$ and $\rho=1 \mathrm{~g} / \mathrm{cm}^{3}$ is the density of the film. This approach assumes a complete surface coverage, where the presence of trapped buffer has been diminished to occupy only the void spaces between densely packed vesicles (the $-\Delta D / \Delta f$ ratio is close to zero and the $-\Delta f$ intercept values were the same on the extrapolation of a linear regression) (Tellechea et al., 2009; Reviakine et al., 2012; Olsson et al., 2013). Figure 3 
302

303

304

305

306

307

308

309

310

311

312

313

314

315

316

317

318

319

320

321

322

323

324

325

326

327

displays the extrapolated Sauerbrey thickness for DOPC SUVs adsorbed onto $\mathrm{SiO}_{2}$ and $\mathrm{Au}$ surfaces. The respective effective frequencies read $h\left(\mathrm{SiO}_{2}\right)=30 \pm 2 \mathrm{~nm}$ and $\mathrm{h}(\mathrm{Au})=62 \pm 5 \mathrm{~nm}$. If one compares these values with the original vesicle diameter in bulk measured by DLS ( $d=79 \pm$ $20 \mathrm{~nm}$ ), vesicles were deformed to a greater extent onto $\mathrm{SiO}_{2}$ surfaces. A more exact approach based on a hydrodynamic model was recently introduced by Cho and co-workers (Gillissen et al., 2017) to estimate the deformation of small vesicles at low surface coverage. The model enables to estimate adsorbed vesicle shape and bending energy by using hydrodynamic spectroscopy (frequency shifts at several overtones). It treats vesicles as ellipsoids and relates the so-called QCM-D force to the scaled viscous penetration depth $\delta / a$ with $\delta$ the viscous penetration depth, and $a$ the non-deformed vesicle radius, in order to probe the adsorbed particle aspect ratio. Further details on the model can be found in (Gillissen et al., 2017).
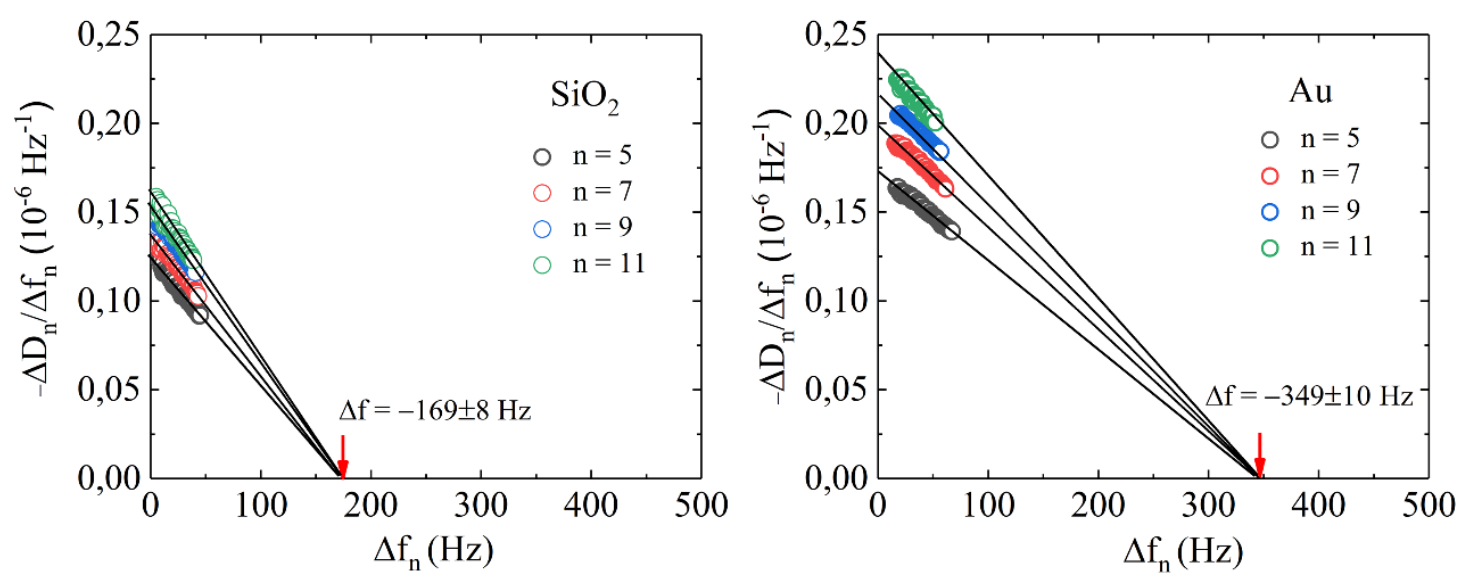

Figure 3. $\Delta D_{n} /-\Delta f_{n}$ ratio as a function of frequency at different overtones for DOPC vesicles adsorbed onto $\mathrm{SiO}_{2}$ and $\mathrm{Au}$.

\subsection{Atomic Force Microscopy (AFM)-based methodology}

Classical structural techniques such as X-ray, NMR or electron microscopy rely on ensemble averaging of molecular properties. Yet, by these means, the underlying molecular dynamics can be hidden, since the signal comes from the average of many unsynchronized molecules in the bulk. The picture changes when using single-molecule techniques. The measured signal of one or a few-molecules reflects the stochasticity of thermally induced processes when randomly crossing free-energy barriers. Single-molecule optical microscopy techniques are particularly well suited for the study of the dynamics of molecules. However, these latter methods rely on the detection of fluorescent markers attached to proteins or lipids, which means that the resolution is limited to $\sim 200 \mathrm{~nm}$ due to diffraction of light and to a few tens of $\mathrm{nm}$ thanks to super-resolution techniques. The atomic force microscopy (AFM) was since its invention in 1986 (Binnig et al., 1986) quickly positioned among the single-molecule, high-resolution structural 
analysis techniques. It provides information concerning structure, function-related conformational changes and supramolecular assemblies, crucial for a complete understanding of biological processes. Nowadays, AFM is in its thirties and has become an invaluable tool for studies at the micro- and nanoscale. As a stand-alone, high-resolution imaging technique and force transducer, it is today an established methodology among the biophysical community. The AFM uses a nanometer-sharp tip -resembling a record player needle- attached to a (micro)cantilever to sense the sample surface while scanning it using piezoelectric elements. An optical system is used to detect the cantilever deflection (or force), thanks to a laser diode beam reflected on the back of the cantilever and detected by a photodiode. Changes in deflection due to sample protrusions while the tip scans the sample are translated into electrical signals thanks to a feedback mechanism. This is schematically represented in Figure 4.

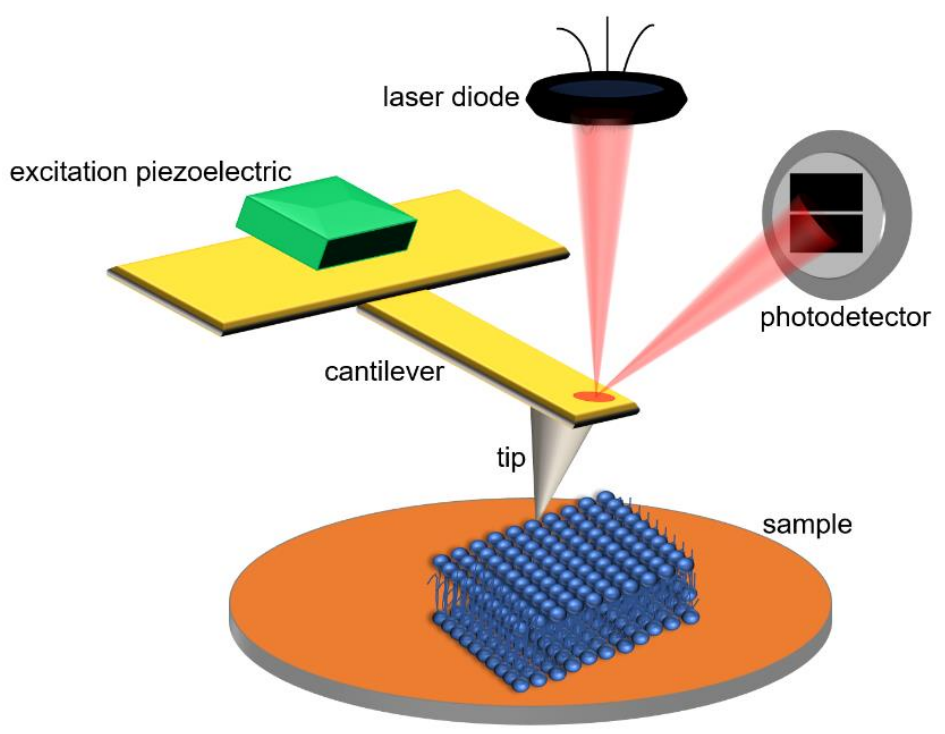

Figure 4. Schematic representation of an AFM instrument. The deflection of the cantilever in the normal direction is monitored by a photodetector which collects the reflection of a laser beam focused at the back of the AFM cantilever. In the tapping mode, thanks to a piezoactuator, the AFM cantilever is excited near its resonance frequency.

One of the main strengths of AFM relies on the possibility of working in a controlled environment (medium composition and temperature) and with great spatial resolution. AFM-imaging mode provides submolecular resolution on a large variety of biological samples: from single molecules, that is, DNA or proteins, to supramolecular assemblies such as SLBs, or even entire cells (Giles et al., 1993)(Dufrêne et al., 2017; Parot et al., 2007). Therefore, AFM has become a wellestablished technique for imaging the topography of lipid membranes that show homogeneous or phase-separated morphology, permitting then to increase the bilayer complexity, from 
351

352

bilayers of one component to multicomponent ones (El Kirat et al., 2010; B. Gumí-Audenis et al., 2016a; Morandat et al., 2013; L Redondo-Morata et al., 2014).

For the study of biological samples, it is common to use AFM-imaging dynamic modes that have an intermittent tip-sample contact. Among them, the most often used is oscillation or tapping mode, in which the AFM cantilever is excited near its resonance frequency. The resulting oscillating tip is intermittently contacting -tapping- the surface, giving rise to a damped oscillation amplitude. Additionally, the resonance frequency is also shifted due to the probesurface interaction. The topography of the surface is reconstructed by monitoring the amplitude of the oscillating cantilever, which is kept constant by adjusting the z-piezo position through a feedback loop. While scanning in the $X-Y$ direction, the amplitude of the cantilever oscillation is kept constant thanks to a feedback control. However, biomolecules are dynamic in essence; hence, to understand how biomolecules work it was only natural to think about increasing the temporal resolution of conventional AFMs. The first time that the concept of High-Speed (HS) AFM was mentioned -to our knowledge- was in 1991 by Barrett and Quate (Barrett, 1991), who did a fairly attempt with the technology at that time. Other research groups, as Paul Hansma's, would challenge the practical speed limitations. It was only in 2010 when the laboratory leaded by Toshio Ando in Kanazawa University (Japan), filmed individual myosin molecules walking on an actin filament (T Ando et al., 2008), operating their AFM instrument at a speed about 1000 times faster than the conventional instruments of that time. Besides the visual impact and scientific insight of those movies, these experiments illustrated that HS-AFM could obtain concomitantly structural and dynamic data, providing insights inaccessible by any other method (Toshio Ando, 2017; Chiaruttini et al., 2015; Kodera et al., 2010; Mierzwa et al., 2017). The basic principles, advantages and limitations of the most common AFM bioimaging mode are nicely detailed in a recent review from Dufrêne et al. (Dufrêne et al., 2017).

Following the capability of the AFM for mechanical manipulation, to sense and apply small forces accurately ( $\mathrm{pN}-\mathrm{nN}$ range), it rapidly developed into an excellent technique to study interactions -inter and intramolecular forces- at the molecular level, the so-called AFM-based force spectroscopy (AFM-FS). The manipulation of single molecules (single molecule force spectroscopy) has led to fascinating new insights in the mechanics of proteins, polysaccharides, synthetic polymers, and DNA at the molecular level (Florin et al., 1994)(Lee et al., 1994)(Radmacher, 1997)(Noy et al., 1997)(Clausen-Schaumann et al., 2000)(Rief et al., 1997)(Hugel \& Seitz, 2001)(P E Marszalek et al., 1999; Piotr E Marszalek et al., 2001)(Giannotti \& Vancso, 2007). 
In the case of lipid bilayers, AFM offers the unique opportunity to probe local physical and mechanical properties, determining the interaction forces with nanoscale lateral resolution, thereby providing new insights into membrane molecular mechanisms. For the nanomechanical characterization with AFM, an SLB is generally first imaged and then a series of force-distance curves is recorded over the same area. In each force-distance curve, the AFM tip away from the surface is approached and retracted at constant velocity while the cantilever deflection is recorded as a function of the $Z$ piezo position. Each portion of the force-distance curve provides information about the physical and mechanical properties of the bilayer, schematized in Figure 5. Upon mechanical contact during approach, the cantilever deflection increases and the SLB is elastically compressed by the AFM probe until the tip suddenly breaks through the SLB, jumping into a contact with the substrate (Figure $5, \mathrm{~b}$ ). The first part of the curve, corresponding to small elastic deformation upon compression, can be used to estimate elastic descriptors of the membrane, like the Young modulus $(E)$, the area compression-expansion modulus $\left(K_{A}\right)$ and bending modulus $\left(k_{c}\right)$ (Dufrêne \& Lee, 2000; Picas et al., 2012; L Redondo-Morata et al., 2016). When performing very small elastic deformations (far from the bilayer rupture) it is also often used for this calculation the retraction part of the curve. To estimate the elasticity of the lipid film, the most common model used is the Hertz model, where the surface is approximate to an infinitely half-space of an isotropic elastic solid and the indenter as non-deformable. The parameter describing the sample is the Poisson's ratio $(v)$, which depends on the nature of the material. For soft biological samples, the Poisson's ratio is generally set to 0.5 (as for incompressible materials). The geometry of the indenter determines the contact area. The original Hertz model considers the shallow contact between two spherical bodies, but several extensions were made for different indenter geometries (D. C. Lin et al., 2007). Again, when performing experiments with small deformations far from the onset of the lipid bilayer rupture, the hysteresis found between the approach and the retract curves is considered as dissipation work and can be used to obtain intrinsic viscoelastic parameters of the viscoelastic material, by the adaptation of common viscoelastic models as power-law rheology or the Kelvin-Voigt model (Garcia et al., 2020).

Moreover, different types of short-range interactions can be measured -DLVO, hydration or steric forces-, for further information review here (Lorena Redondo-Morata et al., 2012). 


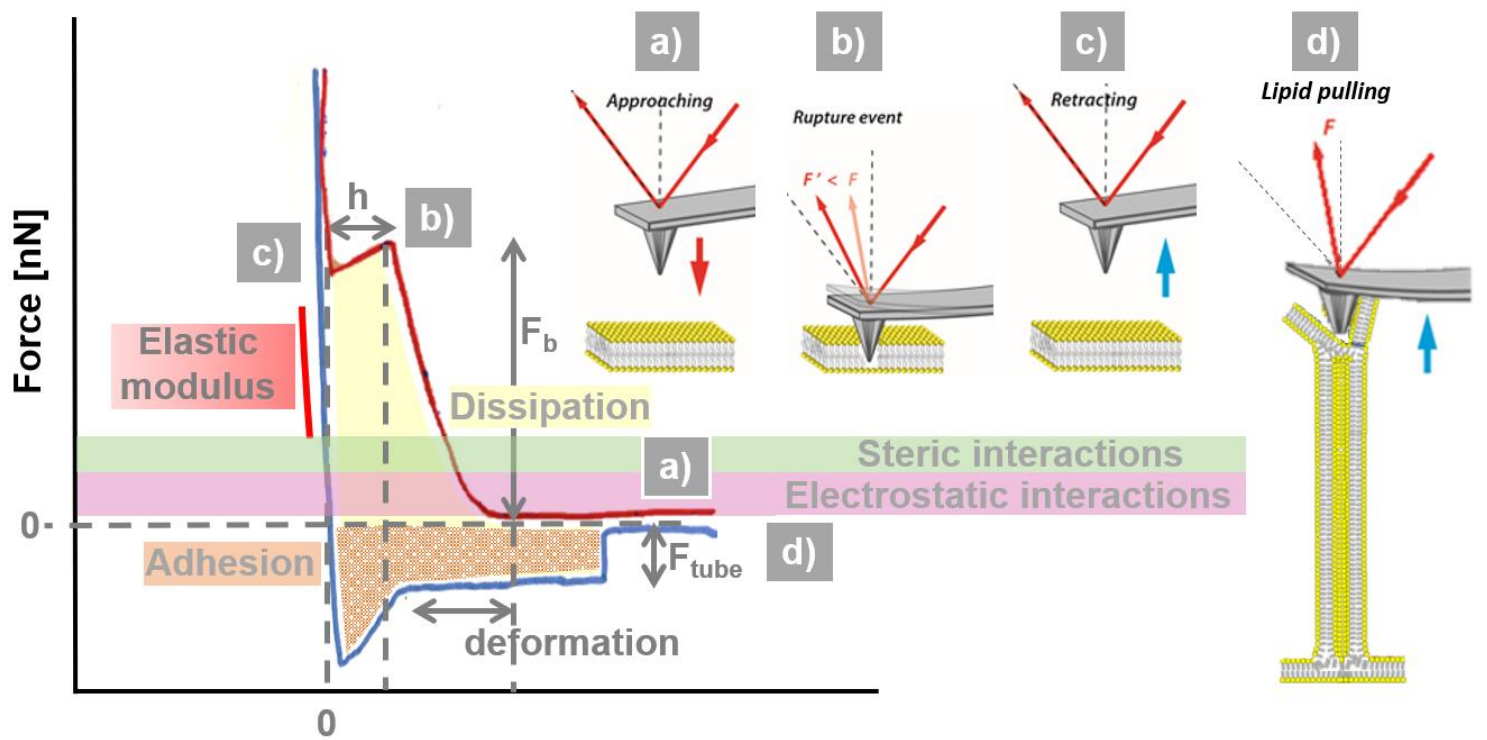

414

415

416

417

418

419

420

421

422

423

424

425

426

427

428

429

430

431

432

433

434

435

436

\section{Tip-sample separation [nm]}

Figure 5. Schematic representation of a representative force curve typically plot in vertical force vs. tip-sample separation. The red curve represents the tip approaching (a). Right after the contact area, short-range tip-sample interactions from different nature appear, predominantly electrostatic (highlighted in pink) and steric (highlighted in green) interactions. When keeping the tip velocity constant, elastic deformation of the membrane is followed by a sudden jump (b) which is interpreted as the breakthrough force $\left(F_{b}\right)$ or the maximum force the bilayer is able to withstand before breaking. $F_{b}$ has been extensively used as a hallmark of the bilayer stability, some of these related works are reviewed in this chapter. The AFM tip reaching the hard substrate underneath is represented as a vertical in the force-separation curve. From the distance in the curve between the breakthrough force and the substrate it can be estimated the minimal bilayer thickness (h) (fully compressed). Afterwards, tip retracts at constant velocity (c). Elastic modulus is usually estimated from the slope of the curve which displays membrane deformation. It is often observed a hysteresis between the approach and retract curves (yellow area) which is related to dissipation work and therefore to the viscoelasticity of the material. During retraction, negative force values are used to estimate the adhesion work between the sample and the AFM tip. Sometimes, a force plateau when retracting the AFM tip away from the SLB can be observed. It characterizes a tube pulling process $\left(c^{\prime}\right)$, defined by the tube growing force $\left(F_{\text {tube }}\right)$ until eventually the AFM tip completely detaches.

The penetration of the AFM tip through the bilayer appears as a discontinuity in the approaching force-separation curve (Figure 5, b). The vertical force at which this discontinuity happens corresponds to the maximum force the bilayer is able to stand before breaking and is defined as the breakthrough force $\left(F_{b}\right)$ or yield threshold force, first observed in the end of the 90 's by 
Dufrêne et al. (Dufrene et al., 1997, 1998)(Dufrêne \& Lee, 2000; S Garcia-Manyes \& Sanz, 2010;

438

439

440

441

442

443

444

445

446

447

448

449

450

451

452

453

454

455

456

457

458

459

460

461

462

463

464

465

466

467

B. Gumí-Audenis et al., 2016a; L. Redondo-Morata et al., 2012; Schneider et al., 2000). $F_{\mathrm{b}}$ is generally of several nanonewtons $(\mathrm{nN})$ and it is considered as a direct measurement of the lateral interactions within the lipid bilayer, at a specified loading rate. From the step in the separation before and after the breakthrough, the SLB thickness at different compression forces (and at zero force) can be extracted.

The mechanical rupture of lipid bilayers is of thermal fluctuation nature, whose destructive action is facilitated and directed by the application of the external force. The penetration of the AFM tip through the SLB has been modeled and conceived as a two-state process with an associated energy barrier (Butt \& Franz, 2002; Franz et al., 2002; Simona Loi et al., 2002). Specifically, two models describe the activation process. The first is the continuum nucleation model, which considers the bilayer as a molecular thin homogeneous film -a two-dimensional fluid layer- between the solid substrate and the solid surface of the AFM tip. The second model ponders the molecular nature of the lipid bilayer and proposes that each molecule in the SLB has specific binding sites corresponding to energetically favorable positions. These sites are energetically equivalent, and as the SLB is pressed by the AFM tip, the energy of the molecules significantly increases, leading them to jump apart and create a hole under the tip. After a critical number of molecules have jumped out of the contact area, the tip indents the SLB due to the high pressure of the remaining molecules, breaking the bilayer. Determining the energy barriers, represented by the Arrhenius law, governing the lipid membranes rupture process contributes to the understanding of the extent of the lateral interactions in the bilayer, and this is generally achieved by following the variation of the $F_{\mathrm{b}}$ with the loading rate $(r)$ by varying the tip-sample approaching velocity $(v)$ in what is called the dynamic force spectroscopy (DFS). Out of equilibrium, the $F_{\mathrm{b}}$ increases linearly with the logarithm of $r$ (and of $v$ as well) (Butt \& Franz, 2002; S Loi et al., 2002). The loading rate dependence with the thermomechanically activated nature of the bilayer rupture kinetics leads to the evaluation of the activation energy of the bilayer failure in the absence of an external force $\left(\Delta \mathrm{E}_{0}\right)$. The activation energy $\Delta E$ for the formation of a hole in the bilayer that is large enough to initiate rupture and lead the tip breakthrough can then be calculated following the expression proposed by Butt et al.:

$$
\Delta E\left(F_{b}\right)=-k_{B} \cdot T \cdot \ln \left[\left(\frac{0.693 \cdot k_{S}}{A}\right) \cdot \frac{d v}{d F_{b}}\right]
$$

where $k_{\mathrm{B}}$ is the Boltzmann factor, $\mathrm{T}$ is the absolute temperature, $k_{\mathrm{s}}$ is the cantilever spring constant, $A$ is the frequency at which the AFM tip attempts to penetrate the bilayer, generally 
approximated to the resonance frequency of the cantilever, $v$ is the tip velocity, and $F_{\mathrm{b}}$ the breakthrough force.

When retracting the AFM tip away from the SLB, it sometimes remains connected to the bilayer through a lipid tube (Figure 5, d). While the tip moves further away, the membrane tube grows longer until it breaks at a certain distance, and the cantilever returns to the equilibrium position. This tube pulling process occurs at constant force, the tube growing force $\left(F_{\text {tube }}\right)$ (Armond et al., 2011; Berta Gumí-Audenis et al., 2018; Maeda et al., 2002; Ralf P Richter \& Brisson, 2003), and it is observed as a force plateau in the retract part of the force-separation curve (Figure 5), at several tens of $\mathrm{pN}$ from which $F_{\text {tube }}$ and the tube growing distance $(d)$ can be recorded. Pulling lipid tubes with an AFM tip out of SLBs is a simplified but analogous situation to some biological processes involving mechanical and conformational adjustments -bending, vesiculation, tubulation-like formation of membrane tethers, endocytic vesicles, etc. (Schmidtke \& Diamond, 2000; Shao et al., 1998; Sheetz, 2001; van Meer et al., 2008). Separation of a membrane segment from the cytoskeleton as well as strong membrane bending are both involved, for which the membrane chemical composition and physicochemical properties are key players. Pulling tethers from cells with an AFM probe is a widely explored approach to evaluate the membrane mechanics on entire cells (Brochard-Wyart et al., 2006; Marcus \& Hochmuth, 2002; Nawaz et al., 2015; Sun et al., 2005). Recently, it has also been established as a method to locally probe SLB mechanics and the contribution of the underlying substrate to the measured properties on SLBs (Berta Gumí-Audenis et al., 2018).

When pulling tethers from cells, the $F_{\text {tube }}$ depends on the membrane properties like bending stiffness $\left(k_{c}\right)$ and in-plane membrane tension $(\sigma)$, and also on the adhesion between the membrane and the cytoskeleton (Sheetz, 2001). In regions where the membrane has separated from the cytoskeleton -bleb or free membrane-, as there is no direct interaction with the cytoskeleton, $F_{\text {tube }}$ strictly relies on $k_{c}$ and $\sigma$ (Dai \& Sheetz, 1999). However, the cytoskeleton adhesion and $\sigma$ are difficult terms to separate, and therefore the concept of apparent membrane tension, $\sigma_{\text {app }}$, has been proposed to include the adhesion energy parameter $\gamma$ (Sheetz, 2001), $\sigma_{\text {app }}=\sigma+\gamma$. When the lipid tube grows under thermodynamic equilibrium, at the limit of zero velocity (static thermodynamic analysis), the following mathematical expression relates the membrane parameters mentioned above with the $F_{\text {tube }}$ (Armond et al., 2011; Canham, 1970; Daillant et al., 2005; E Evans \& Yeung, 1994; Berta Gumí-Audenis et al., 2018; Hochmuth et al., 1996; Marcus \& Hochmuth, 2002; Roux, 2013): $\sigma_{a p p}=\frac{F_{t u b e}{ }^{2}}{8 k_{c} \pi^{2}}$. 
500

501

502

503

504

505

506

507

508

509

510

511

512

513

514

515

516

517

518

519

520

521

522

523

524

525

526

527

528

529

530

531

532

The plus of this approach, AFM-based pulling lipid tubes from SLBs, is that it combines the advantage of the AFM local probing with nanoscale lateral and force resolution, with the simplicity of the SLB model preparation. Besides, the local nanomechanical properties of SLBS can be explored through the combination of the tubing force spectroscopy approach and the $F_{\mathrm{b}}$ analysis exposed before, within the same experimental procedure (Berta Gumí-Audenis et al., 2018).

Although it will not be extensively discussed here, we find important to mention that the constant force approach can also be used to evaluate the membrane rupture kinetics in SLBs and supported lipid multibilayers (SLMs). The AFM tip is approached at constant velocity, and then compresses the membrane at a constant force (force clamp, FC). Through measuring the time it takes to penetrate the bilayer -time to breakthrough $\left(t_{b}\right)$ - distribution at different compression forces, the AFM-FC mode allows to estimate the rate of bilayer failure and calculate the location of the energy barrier maximum -distance to the transition state- along the reaction coordinate $(\Delta \mathrm{x})$ and the activation energy $\left(E_{0}\right)$ that characterize the energy barrier (L RedondoMorata et al., 2012a; Relat-Goberna et al., 2017). Both $E_{0}$ as well as $\Delta x$ were found to be higher for SLBs than for supported lipid multibilayers, endorsing previous suspicion that the mechanical properties of lipid bilayers obtained from nano-indentation are strongly influenced by the underlying hard substrate.

\section{Phase behavior and nanomechanics. From one component membranes to higher complexity}

\subsection{One-component membranes}

\subsubsection{The gel and the fluid phase}

To start with the simplest system and increase the complexity in this revision -which does not intend to be exhaustive- consider a one-component phospholipid bilayer, which is thermalized at a temperature far from its $T_{m}$, the temperature of the main phase transition, at which a disordering of the hydrophobic tails happens followed by a sudden increase of the lateral mobility. We are then able to distinct two different main phases, gel-like or solid ordered phase $\left(s_{o}\right)$ below $T_{m}$, and fluid-like phase or liquid disordered $\left(I_{d}\right)$ above $T_{m}$.

A mechanical descriptor of lipid bilayers that shows significant differences between both phases is the bending modulus $k_{c}$. At $\mathrm{T}<\mathrm{T}_{\mathrm{m}}, k_{\mathrm{c}}$ is an order of magnitude larger than at $\mathrm{T}>\mathrm{T}_{\mathrm{m}}$, rendering lipid vesicles quite stiff, while softer and more deformable above $T_{m}$. In the case of SVLs, where probing deformation is not straightforward, QCM-D has proved to be a useful tool to estimate the extent of vesicle deformation upon adsorption onto surfaces. Reviakine and co-workers 
533 (Reviakine et al., 2012) reported deformation measurements of small DMPC vesicles on $\mathrm{TiO}_{2}$

534 above and below $T_{m}$, and experimentally found out that adsorbed vesicle deformation correlates

535 with the bilayer bending modulus. Liposomes in the fluid phase bear a smaller bending modulus

536 and deform to a greater extent.

537 When imaged with the AFM, the SLBs show typically 4-6 $\mathrm{nm}$ of height. The measured height is 538 also influenced by both the elasticity of each bilayer and the particular AFM methodology, i.e. 539 acoustic-modulation "tapping" mode, contact mode, etc, where the representation of the 540 interface of the lipid bilayer changes. In terms of its mechanical properties, whether we are 541 measuring the elastic deformation or the yield threshold force, a one-component SLB yields a 542 monomodal distribution. While the distribution of deformations or breakthrough forces of the 543 bilayer, being a stochastic process, have been often approached to Gaussian distributions, other 544 distributions based on different theoretical frameworks can also be found in the literature, like 545 the models proposed by Franz et al. (Butt \& Franz, 2002) for the two-state activation process of the bilayer rupture, mentioned in section 3.2.

547 In the first place, it could be said that, in a general way, the bilayer thickness depends on the nature of the lipids -solid-like bilayers are usually higher than liquid-like counterparts of similar number of carbons in the hydrocarbon chains. In an $s_{0}$ phase, the hydrocarbon chains are stretched, so the apparent height is greater, and this is observed when imaging SLBs by AFM. Consequently, stretched acyl chains lead to stronger van der Waals interactions between neighboring molecules, which translates into a shorter intermolecular distance between phospholipids, and a greater mechanical resilience, both elastically and upon AFM tip penetration.

For example, let's briefly consider the elastic deformation of DOPC and DPPC, as models of $l_{d}$ and $s_{0}$ SLBs, respectively. The slope of the curve in the contact zone (elastic regime), as we have commented in section 3.2, is related to the elastic modulus of the material. At room temperature, the slope for the DOPC SLB, which is in the fluid phase, is lower than that for the solid DPPC SLB. This indicates that DPPC bears a larger Young modulus than DOPC and thus the membranes in the $s_{0}$ phase are therefore stiffer than in the $l_{d}$ phase. Both slopes are smaller

561 than the one that can be measured on the bare substrate (often assumed as an infinitely stiff material, such as mica).

Besides the evidenced difference in elasticity for the fluid and gel-like membranes, $I_{\mathrm{d}}$ SLBs show in general lower $F_{\mathrm{b}}$ values than $s_{\mathrm{o}}$ SLBs. A good example are homologous series phospholipids; while at room temperature DLPC $\left(T_{m} \sim-2{ }^{\circ} \mathrm{C}\right)$ SLBs break at force values between 2 and $13 \mathrm{nN}$, 
566

567

568

569

570

571

572

573

574

575

576

577

578

579

580

581

582

583

584

585

586

587

588

589

590

591

592

593

594

595

596

597

598

599

according to the ionic environment, DPPC $\left(\mathrm{T}_{\mathrm{m}} \sim 41.5^{\circ} \mathrm{C}\right) \mathrm{SLBs}$ are pierced by the AFM probe, in similar conditions, in the force range that goes from 7 to $30 \mathrm{nN}$ (Lorena Redondo-Morata et al., 2012). It is also noticeable that the $F_{\mathrm{b}}$ increase with the logarithm of the loading rate is lower for the fluid than for the gel membranes (Lorena Redondo-Morata et al., 2012). This can be expected, if we consider the Bell-Evans or assimilable model, the most likely breaking force depends linearly on the logarithm of the loading rate $r, F(r)=\left(\frac{k_{B} T}{x}\right) \ln \frac{r x}{K_{0} k_{B T}}$, where $k_{\mathrm{B}}$ is the Boltzmann constant, $x$ is the distance to the transition state and $K_{0}$ is the off-rate constant at 0 force (E Evans \& Ritchie, 1997). In this case, $x$ is directly related to the distance between neighboring phospholipid molecules. This intermolecular distance, therefore $x$, is smaller in $s_{0}$ than in $l_{\mathrm{d}}$.

Early work showed how AFM-FS is able to explore quantitatively the molecular determinants that provide mechanical stability to the supported membrane (S Garcia-Manyes, Oncins, et al., 2005a; Sergi Garcia-Manyes et al., 2010). By systematically and independently changing the chemical composition of the lipid headgroup, the length of the hydrophobic tail, and the number of chain unsaturations, \{Citation\} it was possible to describe the mechanical stability of each bilayer as a direct signature of membrane lateral organization (S Garcia-Manyes et al., 2010). The distribution of $F_{\mathrm{b}}$ values as a function of the length of the hydrophobic chain showed that on average, increasing each hydrocarbon tail by 2 extra $-\mathrm{CH}_{2}$ - groups results in $\sim 7 \mathrm{nN}$ increase of the $F_{\mathrm{b}}$, equivalent to an enthalpy increase by roughly $2 \mathrm{~kJ} / \mathrm{mol}$ (Berg et al., 2002). In the same study, the mechanical properties of the linear DPPC were compared with its analogous branched phospholipid, DPhPC, to investigate the effect of acyl chain branching. The distribution of $F_{b}$ showed a decrease from $\sim 20 \mathrm{nN}$ to $\sim 12 \mathrm{nN}$, revealing an important contribution of the lateral $\mathrm{CH}_{3}$ groups on the molecular packing of the bilayer. This observation can be rationalized in terms of the larger area per lipid for the branched DPhPC $\left(76.8 \AA^{2}\right)$ compared to that for the linear DPPC $\left(62.0 \AA^{2}\right)$, as revealed by molecular dynamics simulations conducted in the fluid phase (Shinoda et al., 2007). The nanomechanical effect of the -cis double bonds present in the acyl chains was also investigated. The $F_{\mathrm{b}}$ values distributions for each particular bilayer showed an inversely proportional trend between mechanical stability and number of unsaturations. It was estimated that upon introduction of one additional unsaturation in each acyl chain, $F_{\mathrm{b}}$ values decrease by $\sim 5 \mathrm{nN}$. Interestingly, introducing an asymmetric unsaturation resulted in an even further mechanical destabilization. The resulting molecular tilt reduces the effective packing between molecules, giving rise to a lower $T_{m}$ (Berg et al., 2002). The findings regarding the mechanical implications of the headgroup chemistry were, however, far less straightforward and highly unanticipated. Comparing phospholipids with the same hydrocarbon tails but $-20-1$ 
600

601

602

603

604

605

606

607

608

609

610

611

612

613

614

615

a)

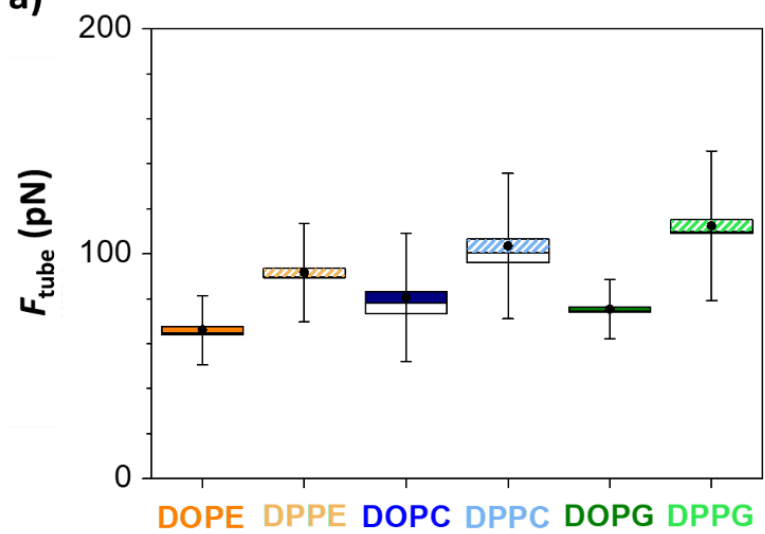
seen in Figure 6b. b)

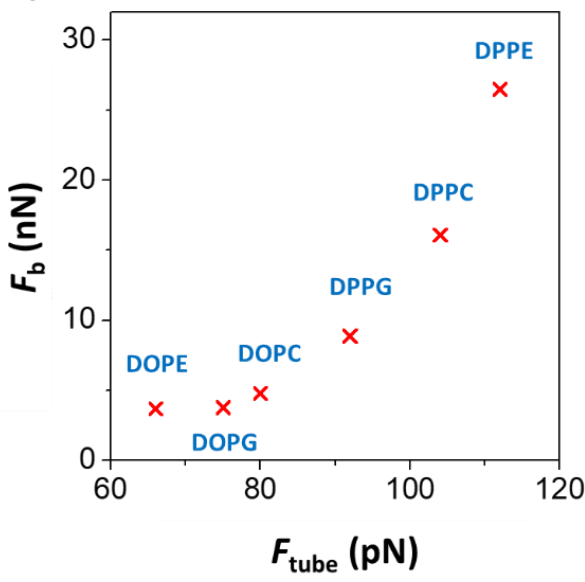

616 different headgroups, a huge range of forces required to indent the bilayer in the same experimental conditions, spanning from $\sim 3 \mathrm{nN}$ (DPPA) all the way up to $\sim 66 \mathrm{nN}$ (DPPG).

Furthermore, the lateral packing differences observed between fluid and gel-like bilayers is also reflected in the tube growing force, pulled as the AFM probe moves away from the SLBs, as this approach has demonstrated to be sensible to the membrane chemical composition in onecomponent SLBs (Maeda et al., 2002)(Berta Gumí-Audenis et al., 2018). Higher $F_{\text {tube }}$ values for DPPC with respect to DMPC have already been observed by Maeda et al. (Maeda et al., 2002) and, as reported in Gumí-Audenis et al. (Berta Gumí-Audenis et al., 2018), $F_{\text {tube }}$ is lower for $I_{d}$ mica-SLBs (in the range of $65-80 \mathrm{pN}$ ) than for $s_{0}$ bilayers of the same headgroup (in the range of 90-110 pN) (Figure 6a). Additionally, while in the $I_{\mathrm{d}}$ state the $F_{\text {tube }}$ slightly augments from PE to PC (66 $\pm 15 \mathrm{pN}$ for DOPE, $80 \pm 25 \mathrm{pN}$ for DOPC and $75 \pm 13 \mathrm{pN}$ for DOPG), it clearly increases when changing the headgroup for so membranes from PE to PC and then PG $(92 \pm 20 \mathrm{pN}$ for DPPE, $104 \pm 30 \mathrm{pN}$ for DPPC and $112 \pm 30 \mathrm{pN}$ for DPPG). Hence, $F_{\text {tube }}$ is generally higher for $s_{0}$ than for $I_{d}$ and goes along with the lateral packing associated to different phospholipid headgroups, in a way that is comparable to the trend observed on the $F_{\mathrm{b}}$ approach, as can be

617 Figure 6. AFM-pulling lipid tubes sensible to the membrane composition in one-component SLBs.

618 a) $F_{\text {tube }}$ values for single component SLBs of different composition (DOPE, DPPE, DOPC, DPPC,

619 DOPG and DPPG). Boxchart for $F_{\text {tube }}$ values show the mean (•), SE (box) and SD (bars). b)

620 Correlation between $F_{b}$ and $F_{\text {tube }}$ values for single component SLBs. The individual phospholipids 621 contain a constant tail length of $18 \mathrm{C}$ with 1 unsaturation (DOPE, DOPG and DOPC) or $16 \mathrm{C}$ and 622 fully saturated (DPPE, DPPC and DPPG), in fluid and gel state at room temperature (RT), 623 respectively. All the measurements were performed in $150 \mathrm{mM} \mathrm{NaCl}, 20 \mathrm{mM} \mathrm{MgCl}, 20 \mathrm{mM}$ 
HEPES (pH 7.4) buffer solution and at RT. Adapted from Ref. (Berta Gumí-Audenis et al., 2018) with permission from the Royal Society of Chemistry.

Taken together, force spectroscopy experiments on SLBs are in qualitative agreement with the literature that correlates the physicochemical properties of membranes with the chemical composition of their phospholipid hydrophobic chains (Rawicz et al., 2000). Noteworthy, thermodynamic quantities such as the melting temperature or the order parameter can be translated into the realm of mechanical stability. The order parameter is usually calculated from NMR-based measurements and provides information about the overall membrane order and more particularly the conformations that the atoms within the lipid tails adopt (Piggot et al., 2017).

\subsubsection{The thermal transition}

636 A useful application of QCM-D is the detection of thermotropic phase transitions within SLBs and SVLs, with the asset that it is label-free, it does not require long temperature equilibration times nor a large amount of sample. When temperature is varied in the absence of any transition, $\Delta f$ and $\Delta D$ typically show a regular, linear temperature dependence, as a reflection of regular, temperature-dependent changes of the viscosity and density of the surrounding liquid medium. When approaching the main phase transition temperature, both frequency and the dissipation responses will differ from a regular temperature dependence, displaying anomalies that reveal that structural changes are taking place (Losada-Pérez et al., 2014; Neupane et al., 2018). Figure 7 displays changes in the frequency and its derivative of an SVL of DMPC of vesicles of around $130 \mathrm{~nm}$, with main transition temperature $\mathrm{T}_{\mathrm{m}} \sim 24{ }^{\circ} \mathrm{C}$ in a rather narrow temperature range. The shape of frequency shift curves and its respective temperature derivative are reminiscent of the enthalpy jump and its heat capacity derivative typically obtained from calorimetric measurements (Heimburg, 1998; Losada-Pérez et al., 2014). Dissipation typically shows jumps and minima in the same temperature range. Therefore, extrema in the first-order derivative of frequency and dissipation curves stand as a token of the layer thermotropic phase transitions. Calorimetry is a state-of-the art technique for the detection of phase transitions

652 based on changes in thermal properties when lipids undergo the main (first-order) transition 653 (heat absorption or release), while QCM-D is based on changes in viscoelastic properties of the adsorbed vesicles as a result of changes in bilayer thickness, rigidity and vesicle shape. Although also detectable in SLBs, the size of the jumps in $\Delta f$ and $\Delta D$ is several orders of magnitude smaller than the size of the jumps observed for SVLs (Wargenau \& Tufenkji, 2014; Hasan \& Mechler, 657 2015). 


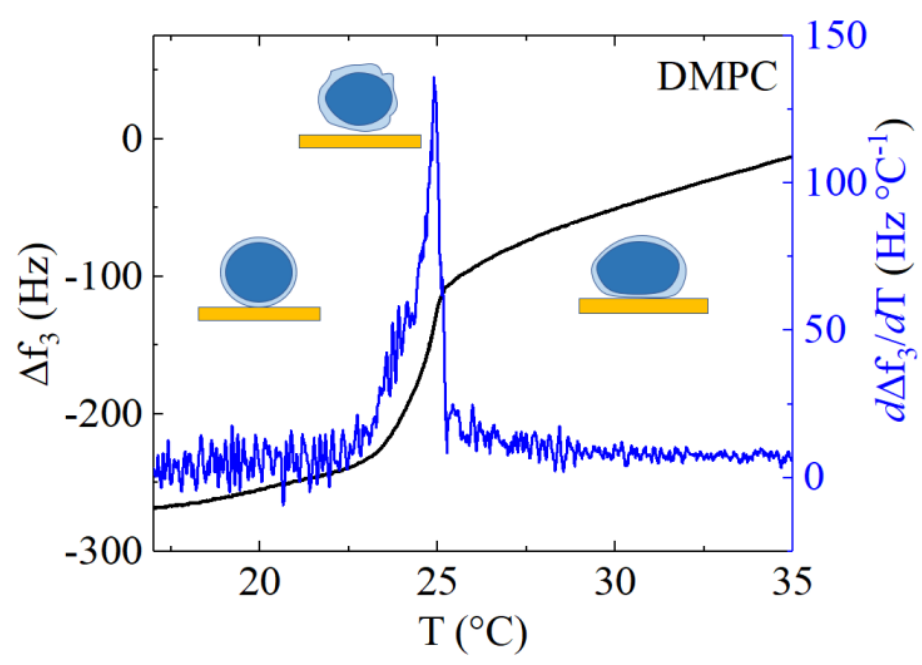

658

659

660

661

662

663

664

665

666

667

668

669

670

671

672

673

674

675

676

677

678

679

680

681

Figure 7. Temperature dependence of the frequency shift $\mathrm{d} \Delta f_{3} / \mathrm{dT}$ (black solid line) and its firstorder derivative (blue solid line) of a DMPC SVL upon heating at $0.2^{\circ} \mathrm{C} / \mathrm{min}$.

Reviakine and coworkers provided a comprehensive interpretation of the mechanisms by which QCM-D detects phase transitions in lipid membranes and the related relative contribution of thickness, stiffness and hydrodynamic channels to the $\Delta f$ and $\Delta D$ fingerprints (Peschel et al., 2016). Upon heating, lipid bilayers in both SVLs and SLBs change from a thicker and stiffer $S_{\text {o }}$ phase to a thinner and less stiff $I_{\mathrm{d}}$ one. Both stiffness and thickness changes in the lipid layer properties across the transition encompass changes in the $\Delta f$ signal (decrease upon heating). In the case of the $\Delta D$, the stiffness and the thickness contributions oppose each other since stiffer layers dissipate less. In the SLBs, this leads to an overtone dependence of the transition peak (Peschel et al., 2016). In thicker and softer SVLs, changes in the shape of the adsorbed vesicles might induce further hydrodynamic channels that contribute to $\Delta D$. As a consequence, the size of the maximum in SVLs is several orders of magnitude larger than the one observed in SLBS.

The thermotropic phase behavior of pure SLBs has been widely related with the (nano)mechanical properties of the bilayer. Combining the previously exposed approaches, the AFM-FS sensitivity to the lipid phase of SLBs is evidenced, positioning it as an appropriate tool to determine the phase thermal transitions. Temperature controlled-AFM has been established as a suitable tool to analyze both the topographical and mechanical evolution of biologically relevant issues that are temperature dependent at the nanometer scale.

Seminal work of Leonenko et al. demonstrated the existence of several phase transitions in DPPC and DOPC mica-supported bilayers (Z. V. Leonenko et al., 2004). Data of both topographical imaging and force measurements provide invaluable insight into membrane behavior during phase transitions. For DPPC SLBs, $F_{b}$ values decrease as temperature increases, and beyond the 
682

683

684

685

686

687

688

689

690

691

692

693

694

695

696

697

698

699

700

701

702

703

704

705

706

707

708

709

710

main transition, DPPC shows similar force plots as the fluid-phase DOPC bilayers. This dependence of the $F_{b}$ on the phase state of the SLB below and above $T_{m}$ has been further reported for different lipids, like DMPC $\left(\mathrm{T}_{\mathrm{m}} \sim 24{ }^{\circ} \mathrm{C}\right)$ that changes $F_{\mathrm{b}}$ from $13-14 \mathrm{nN}$ at $20{ }^{\circ} \mathrm{C}$ below $T_{m}$ to about $6 \mathrm{nN}$ at or $40^{\circ} \mathrm{C}$ DPPC $\left(T_{m} \sim 42^{\circ} \mathrm{C}\right)$ that shows a switch of $F_{b}$ from around 20$25 \mathrm{nN}$ at $27^{\circ} \mathrm{C}$ to around $3-5 \mathrm{nN}$ at $55^{\circ} \mathrm{C}$ (S Garcia-Manyes, Guell, et al., 2005; L Redondo-Morata et al., 2012b). When the DPPC SLB is maintained a temperature slightly above $T_{m}$, coexistence of the gel and fluid phase occurs and a bimodal $F_{\mathrm{b}}$ distribution is obtained (Figure 8), as reported in Gumí-Audenis et al. (B. Gumí-Audenis et al., 2016a). This is because the thermal transitions in SLBs generally happen at temperatures slightly higher and in a broader range than in vesicle suspensions as a consequence of the underlying substrate affecting lateral order and interleaflet coupling (S Garcia-Manyes, Guell, et al., 2005; Z. V Leonenko et al., 2004; L Redondo-Morata et al., 2012b; Seeger et al., 2010). In Garcia-Manyes et al. it was shown that the main phase transition occurs in a range of temperatures of $c a .10-13^{\circ} \mathrm{C}$ (S Garcia-Manyes, Oncins, et al., 2005b).

There is an effect of temperature on the mechanical stability for the SLBs because the increase of temperature directly affects the molecules fluctuations provoking an increment in the area per molecule -lower packing density-, especially noticeable in the gel phase, with the consequent decrease of the $F_{b}$ values. At temperatures around the $T_{m}$ of the lipid, and for a temperature range of a few ${ }^{\circ} \mathrm{C}$, a pronounced drop in $F_{\mathrm{b}}$ takes please, as the SLB gradually transitions to the fluid phase, thereby softening. Several degrees over the $T_{m}$, the $F_{b}$ values are on the order of the ones typically observed for $I_{d} S L B s$ at room temperature (Alessandrini \& Facci, 2012; S Garcia-Manyes, Oncins, et al., 2005a; L Redondo-Morata et al., 2012b). This can be seen in the example shown in Figure 8d, where the DPPC mica-SLB shows an $F_{\mathrm{b}}$ value around of 21 $\mathrm{nN}$ at $27^{\circ} \mathrm{C}$ and slight decreases with temperature as the $42-45^{\circ} \mathrm{C}$ range is reached. At higher temperatures, the $F_{\mathrm{b}}$ remains approximately constant at $c a .3 .5 \mathrm{nN}$. The $F_{\mathrm{b}}$-temperature trend displays a break around the $T_{m}$ value of DPPC.
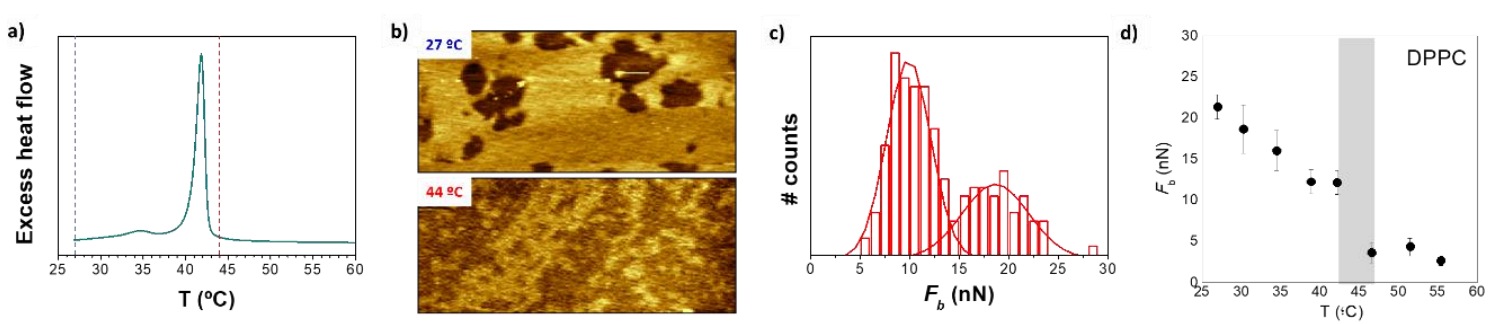

Figure 8. Phase transition of DPPC bilayers: a) DSC thermogram of DPPC vesicle suspension; b) 6 $\mu \mathrm{m} \times 2.5 \mu \mathrm{m}$ AFM images of DPPC silicon-SLB at $27 \stackrel{\circ}{\circ} \mathrm{C}$ and $44 \stackrel{\circ}{\circ} \mathrm{C} ; \mathrm{C}) F_{\mathrm{b}}$ histogram at $44 \stackrel{\circ}{\circ} \mathrm{C}$ and 
711 gaussian fits to the bimodal distribution. All experiments performed in $150 \mathrm{mM} \mathrm{NaCl}, 20 \mathrm{mM}$

$712 \mathrm{MgCl}_{2}, 20$ mM HEPES (pH 7.4) buffer solution. Adapted from Ref. (B. Gumí-Audenis et al., 2016a).

713 d) Mean $F_{b}$ value of DPPC mica-SLB as a function of temperature, in $10 \mathrm{mM} \mathrm{HEPES,} 20 \mathrm{mM} \mathrm{MgCl}$

714 and $150 \mathrm{mM} \mathrm{NaCl}(\mathrm{pH}$ 7.4). The shadowed vertical line marks the observed transition

715 temperature range. Adapted from Ref. (L Redondo-Morata et al., 2012b)

716 Additionally, Mouritsen and coworkers proposed that AFM topography at a varying temperature

717 could decouple the phase transition of the two different leaflets of the mica-supported bilayer

718 (D. Keller et al., 2005). The low temperature transition was proposed to be related to the melting

719 of the leaflet far from the substrate surface, and the second transition to the phase transition of

720 the leaflet in contact with the mica. Such decoupling would respond to the stronger interaction

721 between the lipid headgroups of the proximal monolayer and the mica support.

\section{4.1.2.2. Pretransition}

723 Some lipids present a pretransition before the main first-order transition which gives rise to an

724 intermediate order in the lipid bilayers referred to as ripple phase. Ripple phases are curved

725 structures with undulating surface topography. AFM imaging revealed the structure of the ripple

726 phase in hydrated conditions at the nanometer scale (Kaasgaard et al., 2003), and such particular

727 structure has been recently explored nanomechanically (Majewska et al., 2020). It can be found

728 when solid phase lipids experience an increase in temperature and go into the fluid phase, and

729 alternatively it occurs when the fluid phase is cooled down. The observation of this phase

730 transition process is characterized by a melting point temperature of the ripple phase to fluid

731 phase, nucleation point and growth directionality for the formation of the ripple phase. HS-AFM

732 coupled to a temperature-controlled system can report directly ripple to fluid phase transitions

733 (reversibly) in real time and at high resolution (Takahashi et al., 2016). Transition processes from

734 ripple phase to fluid phase and from ripple phase to metastable ripple phase to fluid phase,

735 could be reversibly, phenomenologically and quantitatively studied. In Takahashi et al., results

736 showed phase transition hysteresis in fast cooling and heating processes, while both melting

737 and condensation occurred at $24.15^{\circ} \mathrm{C}$ in quasi-steady state situation. A second metastable

738 ripple phase with larger periodicity was formed at the ripple phase to fluid phase transition when

739 the buffer contained $\mathrm{Ca}^{2+}$. Thermodynamically, a lipid system containing small domains does not

740 necessarily correspond to a phase coexistence regime, but they may constitute a one phase

741 regime with micro- or nanoscale heterogeneities ${ }^{1}$ (Heimburg, 2000). Altogether, these findings

\footnotetext{
${ }^{1}$ We will further visit this topic in Section 5).
} 
742

may have relevance in more complex cellular systems where lipid nanodomains form and dissociate reversibly.

\subsection{Phase coexistence}

Phase segregation and coexistence in lipid bilayers generally happens in the simplest models when mixing a fluid and a gel-like phospholipids, and in the more complex mixtures, like those containing phospholipids, sphingomyelins, ceramides, and cholesterol. At room temperature the bilayers composed of a mixture of a gel and a fluid phospholipid generally segregate into different domains, showing a continuous fluid phase enriched with $/_{\mathrm{d}}$ lipid and thicker dispersed domains associated to a phase enriched in the $s_{o}$ lipid. When the $T_{m}$ of the gel-phase lipid is overcome, the binary phospholipid system becomes homogeneous and fluid. Among the many that can be found in the literature, we here mention some examples of these binary systems.

The capability of QCM-D to detect lateral phase coexistence and map phase diagrams for binary lipid mixtures of saturated phospholipids will be provided for binary mixtures of homologous phosphatidylcholines DMPC and DSPC, whose chemical structures differ in four methylene groups. This system has been thoroughly investigated experimentally and theoretically in free standing lipid systems (Ehrig et al., 2011; Fidorra et al., 2009; Mabrey \& Sturtevant, 1976). DMPC:DSPC mixing behavior is driven by the large hydrophobic mismatch between the two components and departs from ideality as a consequence of the large difference in $T_{m}$ of the pure constituents.

Figure 9 (left panel) shows representative QCM-D signature of phase transitions we now revisit taking place upon heating pure SVLs of pure DMPC and three DMPC:DSPC mixtures (LosadaPérez, Mertens, et al., 2015). The diameter of the precursor vesicles forming the SVLs was around $130 \mathrm{~nm}$. For pure DMPC, the main transition takes place in a rather narrow temperature range around $\mathrm{T}_{\mathrm{m}} \sim 24{ }^{\circ} \mathrm{C}$. The first-order derivatives of $\Delta f$ curves capture the typical behaviour of two-component lipid mixtures displaying $s_{0}$ and $l_{\mathrm{d}}$ phase coexistence. As very low DSPC concentration (DSPC mole fraction $=0.1$ ), the transition is shifted towards higher temperatures and takes place in a broader temperature range indicating a loss of intermolecular cooperation between DMPC molecules to undergo the main transition due to the presence of DSPC. For the mixtures close to the equimolar concentration two small peaks can be observed indicating the coexistence of two phases, $s_{0}$ and $I_{\mathrm{d}}$. The peaks are not simple additions of the peaks of the individual constituents, the size of each peak scales with the concentration of each lipid in each coexisting phase. For instance, the inset of Figure 9 displays the peaks of the mixture DMPC:DSPC (40:60). The low-temperature peak corresponds to the DMPC-rich phase, while the 
782

high-temperature peak to the DSPC-rich phase. As expected, the size of the latter is higher, since the concentration of DSPC in the mixture is larger.

The analysis of the peaks using the tangent method (Ehrig et al., 2011) to determine the onset and completion temperatures enables to map the phase diagram of this mixture. The solid lines are added as a guide to the eye, delimiting the phase coexistence region, dark red (liquidus line), light blue (solidus line) and follow experimental heat capacity values by Maubrey et al. (Mabrey \& Sturtevant, 1976) obtained for multilamellar vesicles of the same concentration.
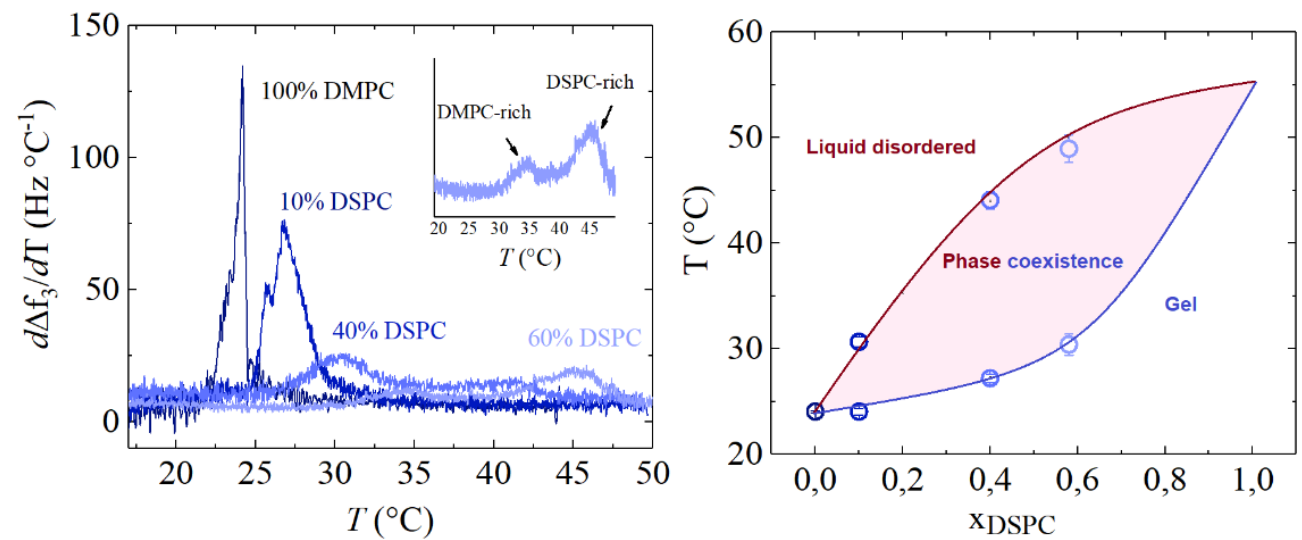

Figure 9. Left panel: temperature profiles of the first-order temperature derivative of the frequency shifts for pure DMPC and DMPC:DPSC mixtures 10\%, $40 \%$ and $60 \%$ mole fraction (in percentage) of DSPC. Right panel: phase diagram obtained from extracting onset and completion temperatures from the peaks (Losada-Pérez, Mertens, et al., 2015).

As discussed in the previous sections, the nanomechanical properties determined by AFM-FS on SLBs are distinctive of the chemical structure and molecular organization of a bilayer at specific environmental conditions. This allows for a direct correlation of the elastic modulus, $F_{\mathrm{b}}$, or $F_{\text {tube }}$ values with the composition in phase-segregated membranes. In most cases, the AFM topographical characterization easily identifies the segregation of an SLB in different domains, due to their difference in thickness and compressibility. However, it is possible to reveal the presence of different domains in an SLB on the basis of their different mechanical properties even when the domains have a height difference which is too small to be detected by conventional imaging.

Bernchou et al. studied, using combined fluorescence microscopy and AFM, the nucleation and growth of domains in equimolar binary SLBs composed by DOPC and DPPC (Bernchou et al., 2009), a mixture that shows coexistence of $I_{d}$ and $s_{o}$ domains at room temperature (Z. V. Leonenko et al., 2004). These authors could observe that during cooling the shape of the domains evolved from compact to a branched morphology, which they discussed as the domain 
801

802

803

804

805

806

807

808

809

810

811

812

813

growth being controlled by the diffusion of DPPC from the liquid phase toward the solid domain interface. In the late stages of the growth, the size and overall shape of the domains depend on the position of the nucleation points relative to the surrounding nucleation point positions.

A mechanical contribution concerning the elastic deformation of the membrane before the breakthrough point should be considered to fully comprehend the force-distance curve. A method to study the elastic properties consists of quantitative nanomechanical mapping (Picas et al., 2012). In (L Redondo-Morata et al., 2016), the equimolar binary lipid mixture DPPC:DOPC was studied, where $I_{\mathrm{d}}$ and $s_{\mathrm{o}}$ coexisting domains in SLBs are large and directly visually discernable by AFM imaging (Figure 10a) with clearly distinguishable heights $(\sim 0.8 \mathrm{~nm}$ height difference, Figure 10b). Elastic modulus nano-mechanical mapping (Figure 10c) shows good agreement with the $E$ values in the literature, characterized mechanically by AFM (Picas et al., 2012; Rawicz et al., 2000) and micropipette aspiration (Dieluweit et al., 2010). The mean $E$ values are $26_{-12}^{+58} \mathrm{MPa}$ for the $I_{d}$ domain and $33_{-15}^{+75} \mathrm{MPa}$ for the $s_{o}$ domain, respectively (Figure 10d).
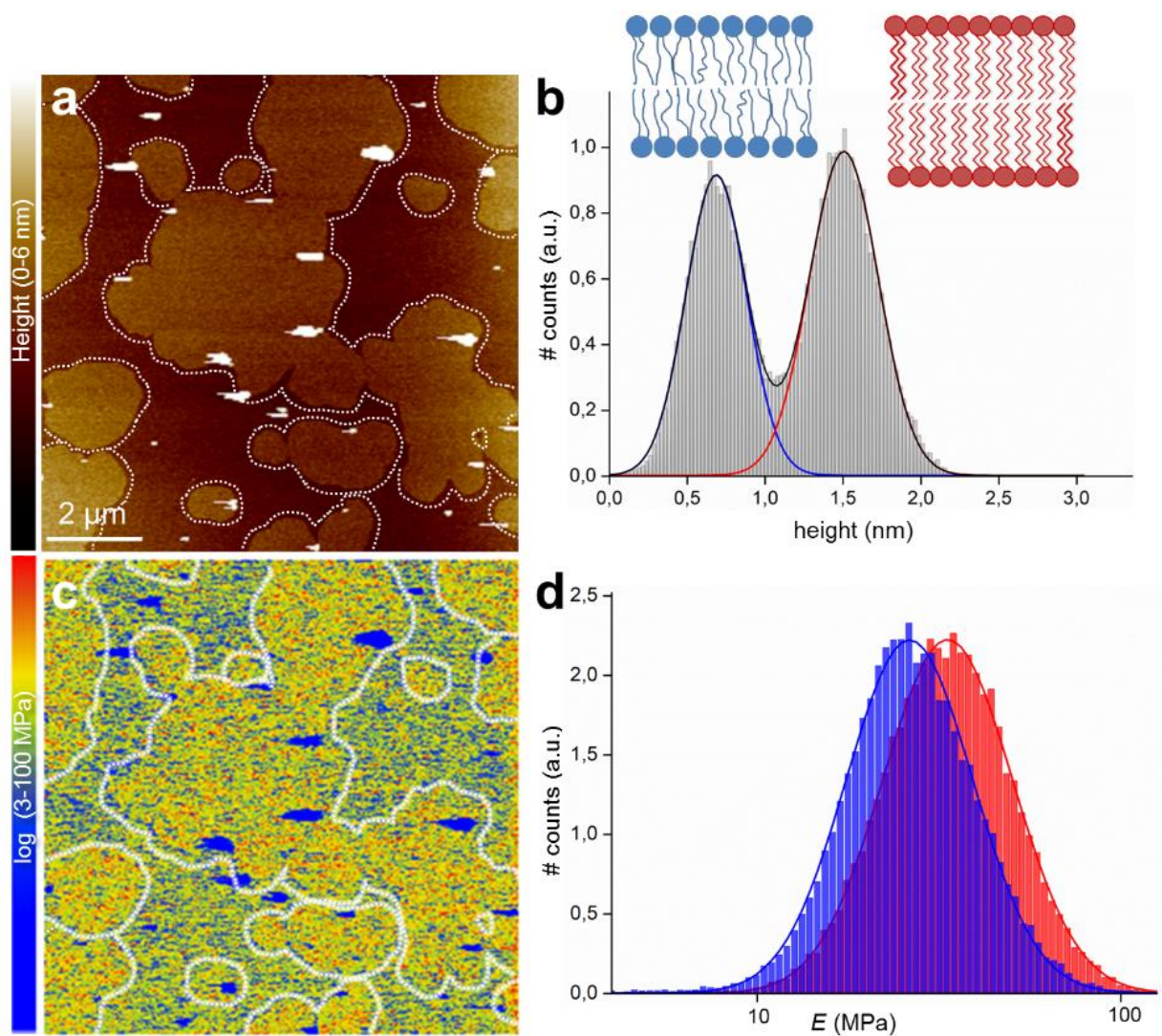

Figure 10. PF-QNM AFM Topography and Elasticity mapping of DOPC:DPPC (1:1) SLBs. a) Topography image ( $\mathrm{nm}$ ) and b) height histogram analysis of the topography (a). The dashed lines in a) represent the domain edges defined by edge detection. The solid lines in b) are Gaussian fits 
and DPPC so (red line) domains of $0.82 \mathrm{~nm}$. c) The corresponding stiffness map (log scale, MPa) and d) Young's modulus values histograms (log scale, MPa) of the regions outlined in the topography (a) and stiffness (c) maps, corresponding to the DOPC (blue) and DPPC (red) domains. The solid lines are Gaussian fits to the distributions peaking at $26_{-12}^{+58} \mathrm{Mpa}$ (DOPC) and $33_{-15}^{+75} \mathrm{Mpa}$ (DPPC) (from reference (L Redondo-Morata et al., 2016) with permission).

Using the Young modulus for each domain of the DOPC:DPPC mixture together with the experimental bilayer thickness $(h)$, the thin shell theory can be used to calculate the area compression-expansion modulus $\left(K_{A}\right)$ and bending modulus $\left(k_{c}\right)$ as (Rawicz et al., 2000 ):

$$
K_{A}=\frac{E h}{\left(1-v^{2}\right)} \quad \text { and } \quad k_{c}=\frac{E h^{3}}{24\left(1-v^{2}\right)}
$$

When lipid domains separate in lipid mixtures, or when lipids surround membrane embedded proteins there is, in general, a hydrophobic mismatch at the domain interfaces (Wallace et al., 2006) or protein/bilayer interfaces (Mouritsen \& Bloom, 1984), with an ensuing compression or stretching of the hydrocarbon chains in the vicinity of the interface. This hydrocarbon tail stretching or compression may influence the distribution of domain sizes and the kinetics of domain separation, as well as membrane protein function. The compression/stretching of the acyl chains of the $s_{o}$ and $l_{d}$ domains can be determined by minimizing the elastic energy of the system, $G_{s}(x)$, expressed assuming volume conservation (Wallace et al., 2006) as

$$
G_{S}=\frac{K_{O} A_{O}}{2 h_{O}^{2}}\left(x-h_{O}\right)^{2}+\frac{K_{D} A_{D}}{2 h_{D}^{2}}\left(x-h_{D}\right)^{2}
$$

where $K_{o}$ and $K_{d}$ are the stretch moduli for $s_{o}$ and $I_{d}$, respectively. $A_{o}$ and $A_{d}$ are the lipid area per molecule for $s_{o}$ and $I_{d}$, respectively. $A$ values are well-documented in literature (notably through X-ray diffraction and Langmuir methods), being $0.72 \mathrm{~nm}^{2}$ for $I_{d}$ DOPC and $0.49 \mathrm{~nm}^{2}$ for $s_{o}$ DPPC at room temperature (Nagle \& Tristram-Nagle, 2000). $h_{o}$ and $h_{d}$ are the measured bilayer thickness of $s_{o}$ and $l_{d}$, respectively. The estimated $G_{s}$ values for the DOPC $l_{d}$ and the DPPC $s_{o}$ domains are $0.27 k_{\mathrm{B}} \mathrm{T}$. Hence, the experimental determination of $E$ allows to estimate the stretch modulus and the bending stiffness of the lipid membrane, as well as the energetic cost of the lipid domains to accommodate to different thickness.

For the SLB system composed of a POPE:POPG mixture (Picas et al., 2009), AFM images revealed the existence of two separated phases, the higher showing a region with protruding subdomains. AFM-FS was then used to clarify the nature of each phase. The values of $F_{\mathrm{b}}$, adhesion force and height extracted from the force curves were assigned to the corresponding $s_{0}$ (the mechanically stable domains) and $l_{d}$ phase (the mechanically labile phase). In Crespo- 
Villanueva et al., while the SLB composed of equimolar DSPC:DOPC was shown to phase segregate, clearly demonstrated in the AFM topographical images and the correlative $F_{\mathrm{b}}$ maps and bimodal distributions around $\sim 40 \mathrm{nN}$ and $\sim 5 \mathrm{nN}$, the equimolar mixture of the two $l_{\mathrm{d}}$ lipids DOPC:DOPS led to a homogeneous topography and narrow $F_{\mathrm{b}}$ distribution around $3.5 \mathrm{nN}$ (Crespo-Villanueva et al., 2018). In Domenech et al., they studied ternary mixtures of POPC:POPE:CL, a minimum system that mimics the mitochondrial inner membrane (Domenech et al., 2007). They could observe laterally segregated domains whose height decreased with increasing temperatures.

Pulling lipid tubes with the AFM probe also allows to characterize phase segregated bilayers and laterally resolve the different domains in multicomponent SLBs (Berta Gumí-Audenis et al., 2018). This is exemplified in Figure 11, a bilayer composed of the $I_{\mathrm{d}}$ lipid DOPE and the $s_{\mathrm{o}}$ lipid DPPG in ratio DOPE:DPPG 25:75, that at room temperature forms SLB patches with coexisting domains: gel-phase rich in DPPG, dispersed in fluid-phase rich in DOPE. When indented by the AFM tip at a constant velocity of $1 \mu \mathrm{m} \mathrm{s}^{-1}$, a bimodal $F_{\mathrm{b}}$ distribution is obtained, with differentiated mean $F_{b}$ values associated with the fluid $(\sim 5 \mathrm{nN})$ and gel $(\sim 12 \mathrm{nN})$ domains. Analyzing the retract force-separation curves that showed the tube pulling event allowed to represent the $F_{\text {tube }}$ distribution and map, where the lower force values (red pixels) are distributed in the peripheral part of the SLB patch and higher forces (yellow pixels) in the center, and the $F_{\text {tube }}$ distribution is resolved when sorting according to the corresponding $F_{\mathrm{b}}$, giving a mean value of $75 \pm 20 \mathrm{pN}$ for the DOPE-rich phase, and $112 \pm 25 \mathrm{pN}$ for the DPPG-rich phase. SLBs allows to infer partial miscibility among them.

a)

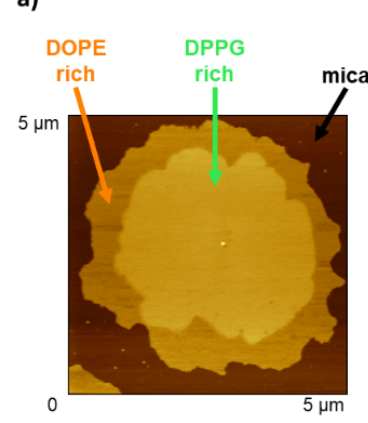

872

873

874 b)

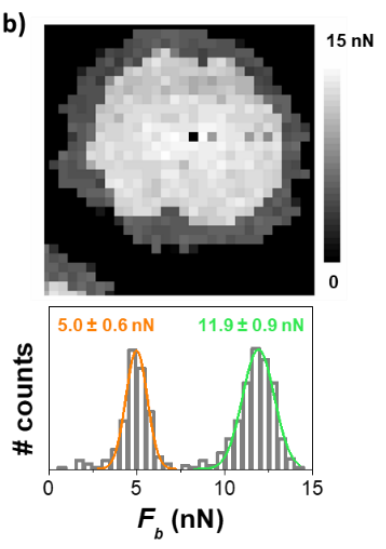

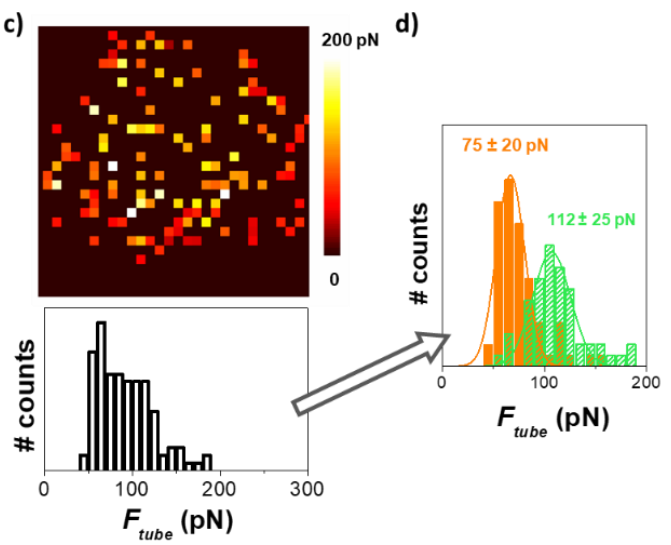

Figure 11. DOPE:DPPG (25:75). a) AC-mode AFM topographical image; b) $F_{\mathrm{b}}$ map and distribution;

c) $F_{\text {tube }}$ map and distribution; d) $F_{\text {tube }}$ distributions resolved by filtering according to the $F_{b}$ 
875

876

877

878

879

880

881

882

883

884

885

886

887

888

889

890

891

892

893

894

895

896

897

898

899

900

901

902

903

904

905

906

907

distribution. Reproduced from Ref. (Berta Gumí-Audenis et al., 2018) with permission from the Royal Society of Chemistry.

Higher complexity models, like the ternary lipid mixtures containing an unsaturated phosphatidylcholine (PC), a saturated PC or sphingomyelin (SM), and Cholesterol (Chol) have been probably the most studied due to the physiological abundance of these lipids. Depending on the ratio of the components, these mixtures have shown to exhibit two coexisting fluid phases: an $I_{0}$ phase that is enriched in Chol and SM or saturated PC, and an $/ \mathrm{d}$ phase that is predominantly composed of unsaturated PC (Johnston, 2007), and the possibility of a coexisting $s_{0}$ phase. The group of Schwille has extensively analyzed the domain organization in DOPC/SM/Chol model membranes by means of AFM in combination with fluorescence correlation spectroscopy (FSC) (S Chiantia, Kahya, et al., 2006; S Chiantia, Ries, et al., 2006; Salvatore Chiantia et al., 2007, 2008) and by performing AFM-FS experiments on the different phases they have shown that the average force needed to pierce the $l_{0}$ phase of the DOPC/SM/Chol bilayer is about $4 \mathrm{nN}$ higher than the one needed to pierce the $l_{\mathrm{d}}$ phase. In a parallel study, Zou and coworkers explored the influence of different cholesterol contents on the morphology and (nano)mechanical stability of phase-segregated DOPC/SM/Chol lipid bilayers by means of AFM imaging and force mapping (R M A Sullan et al., 2010). They observed consistently higher $F_{b}$ values in the SM/Chol-enriched $I_{0}$ domains than in the DOPC-enriched $l_{\mathrm{d}}$ phase, and that the (nano)mechanical stability of both coexisting phases counterintuitively decreased with increasing Chol content. By following the dependence of the $F_{b}$ value with the loading rate in both phases, they calculated the activation energies $\left(\Delta E_{0}\right)$ of bilayer rupture at zero applied force. $\Delta E_{0}$ values obtained were in the range $75-125 \mathrm{~kJ} / \mathrm{mol}$ for all tested Chol contents and did not show a dependence on the different lipid phases.

Besides sphingolipids, ceramides (Cer) have also gained attention in physicochemical studies by means of AFM, since they are known to be predominant in physiologically relevant nanodomains -which have been controversially called for many years lipid rafts (Goñi, 2019a). Longo and coworkers observed that DLPC/GalCer/Chol ternary mixtures only display $l_{\mathrm{d}} / s_{\mathrm{o}}$ coexistence and no $I_{0}$ phase even at high Chol content (Blanchette et al., 2006). By measuring the area/perimeter ratios of the segregated domains, the authors concluded that Chol was able to decrease the line tension between the two phases. In another study (W.-C. Lin et al., 2007), they could also observe a phase coexistence attributed to $I_{\mathrm{d}} / l_{0}$ phases with POPC and DOPC at $10 \mathrm{~mol} \% \mathrm{Chol}$, an indication that fatty acyl chain unsaturation, cholesterol ratio and lipid hydrophobic mismatch in the organization of multi-component lipid systems. 
908

909

910

911

912

913

914

915

916

917

918

919

920

921

922

923

924

925

926

927

928

929

930

931

932

933

934

935

936

937

938

939

940

941

Ceramide incorporation on coexisting fluid phase and ordered domains in phase-separated binary and ternary lipid mixtures was shown to affect the lipid spatial organization in domains, with the appearance of a Cer-enriched gel-like phase and the displacement of cholesterol from rafts (S Chiantia, Kahya, et al., 2006; Salvatore Chiantia et al., 2007; Ira et al., 2009). In order to directly probe and quantify the (nano)mechanical stability and rigidity of the Cer-enriched platforms, Zou et al. performed AFM topographical images and force mapping that allowed the determination of the $F_{b}$ value and elastic modulus of the different phases (Ruby May A Sullan et al., 2009a, 2009b; Zou \& Johnston, 2010). They confirmed the expulsion of cholesterol from sphingolipid/Chol-enriched domains as a result of Cer incorporation, and observed an increased mechanical stability attributed to the influence of Cer in the lipid organization and packing behavior, also reported later on GalCer rich domains on model membranes with PC and Chol (Berta Gumí-Audenis et al., 2015).

The group of Goñi has systematically explored the effect of the acyl compositions of sphingomyelins and the corresponding ceramide counterparts in ternary mixtures with PC and Chol (García-Arribas et al., 2016; González-Ramírez et al., 2019; Jiménez-Rojo et al., 2014)). Combining confocal microscopy, DSC, AFM imaging and FS, they compared the effects of C24:1 Cer (nervonoyl ceramide, nCer) with those of C16:0 Cer (palmitoyl ceramide, pCer) in bilayers composed basically of DOPC, SM (either C24:1, nSM or C16:0, pSM) and Chol (García-Arribas et al., 2017). AFM-FS showed that nCer has a lower stiffening effect than pCer, while the presence of $\mathrm{nSM}$ reduces the stiffness. When the proportion of phospholipid increases beyond $60 \mathrm{~mol} \%$, a lateral phase separation occurs at the micrometer scale (González-Ramírez et al., 2019). The authors interpreted this data as a pCer:Chol interaction, that would predominate at the lower phospholipid concentrations. The putative pCer:Chol complexes (or nanodomains) would mix well with the phospholipid. At higher SM concentrations pSM:pCer and pSM:Chol interactions would become more important, giving rise to the coexisting $s_{0}$ and $l_{0}$ phases, respectively. Heterogeneity, or lateral phase separation, occurs more easily with pSM than with DPPC, indicating a higher affinity of SM over DPPC for Chol or Cer. Altogether, the findings of Goñi and coworkers show the sharp increase in complexity when membranes exhibit different sphingolipids of varying $\mathrm{N}$-acyl chains, which should be a common issue in an actual cell membrane environment.

\subsection{The role of cholesterol}

Cholesterol is a fundamental constituent of the eukaryotic cell plasma membranes. Eukaryotic cell membranes are not entirely in the conventional / phase. Instead, they are, at least partially, in the Chol-rich liquid-ordered $\left(I_{0}\right)$ phase or a phase with similar properties. $I_{0}$ phase is 
942

943

944

945

946

947

948

949

950

951

952

953

954

955

956

957

958

959

960

961

962

963

964

965

966

967

968

969

970

971

972

973

974

975

characterized by a high degree of acyl chain order and is favored by high- $\mathrm{T}_{\mathrm{m}}$ lipids with saturated acyl chains such as sphingolipids, when they are mixed with Chol, as exposed in the previous section. Chol plays the essential function of regulating the physical properties of the cell membrane as it controls the lipid organization and phase behavior, regulates the membrane fluidity and its mechanical strength, influencing the passive permeability of water and other small molecules (Pan et al., 2008; Pinkwart et al., 2019). From the molecular perspective, Chol is considered to produce a condensing effect and induce ordering to fluid phase lipids forming the membrane and to have the opposite effect on phospholipids present in the gel phase (Hung et al., 2007; Rog et al., 2009). However, several experiments pointed out the complex effect of Chol on lipid bilayers, which depends on the molecular structure of the neighboring lipids: the degree of chain unsaturation, the length of the hydrophobic tail and the chemistry of the headgroup (Pan et al., 2008; Mills et al., 2009; Garcia-Manyes et al., 2010). In view of acknowledging the particular role of cholesterol, let us briefly outline results on the phase behavior of SVLS and SLBs consisting of binary mixtures of saturated phospholipids and cholesterol using mechanical descriptors such as shear viscosity of SVLs and nanomechanics of SLBs.

We first describe the phase behavior of SVLs containing binary mixtures of DMPC:Chol using QCM-D. Upon the addition of Chol to phospholipid bilayers, the sharp main phase transition in pure DMPC gradually diminishes and eventually disappears over a given Chol concentration. In the case of DMPC, SVLs showed no transition from $30 \%$ cholesterol. Figure 12 shows normalized shear viscosity $\eta_{\text {norm }}=[\eta(t) / \eta(t=0)$, with $t$ the time] for pure DMPC and DMPC:Chol $(20 \mathrm{~mol} \%$ Chol) calculated using a Voigt-based viscoelastic model (Voinova et al., 1999). At intermediate Chol concentrations, asymmetric peaks can be observed. These peaks encompass two main contributions, a sharp, narrow peak and a broader one that overlaps with the former (see right panel in Figure 12 for a more detailed view including Gaussian fitting). Two domains can be identified, namely, the sharp contribution can be ascribed to the pure DMPC-rich domains undergoing the main transition and the latter to the Chol-rich ones. The broader peak appears as a result of the reduced mobility of the hydrophobic chains of DMPC in Chol-rich domains. The presence and the size of these domains depend strongly on the concentration of cholesterol and the temperature. On increasing the amount of cholesterol, the temperature range of the overall transition increases as a result of the extension of the second broad contribution, while the size of the maximum corresponding to the melting of the single lipid decreases. No phase transition is detected at Chol concentration over $30 \mathrm{~mol} \%$ indicating the formation of a homogeneous liquid ordered $l_{0}$ phase at a macroscopic level. Such a large cholesterol concentration inhibits 
the formation of extended domains. A similar pattern of behavior was observed for DPPC:Chol SVLs, however, for this later system, the completion temperature of the overall transition at intermediate cholesterol concentrations was not attained, due to the (upper) limited temperature range of commercial QCM-D systems (Losada-Pérez, Khorshid, et al., 2015). These results are in agreement with recent calorimetric studies of free-standing membranes of the same systems (Mannock et al., 2010).
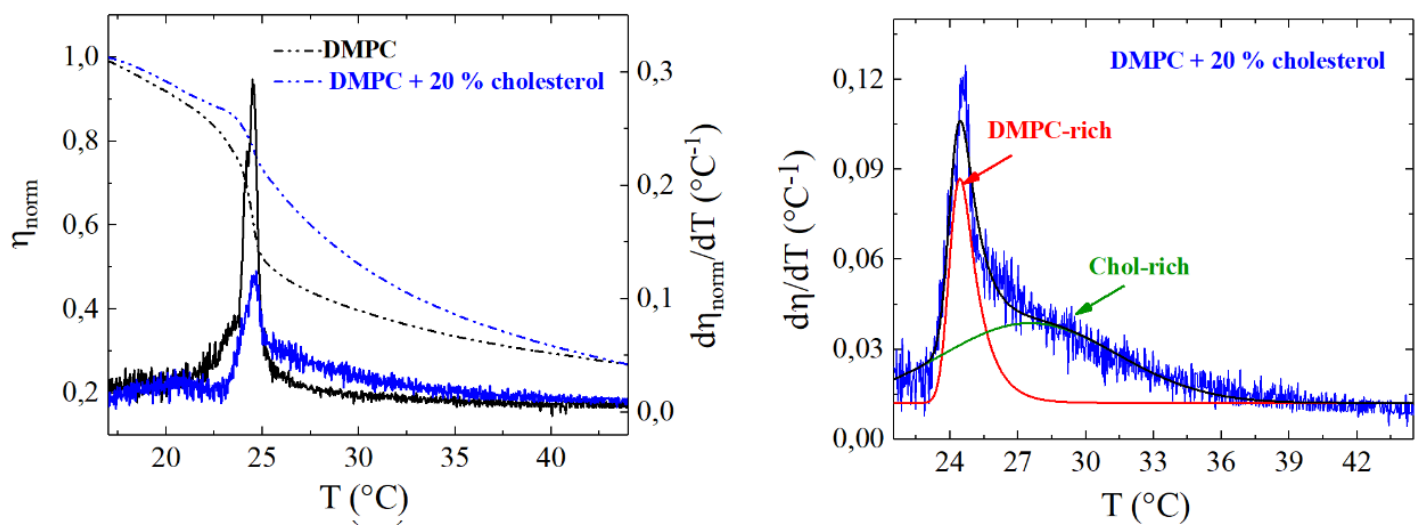

Figure 12. Left panel Temperature dependence of the normalized effective shear viscosity (dash dotted lines) and its first-order derivative (solid line) of DMPC and DMPC $+20 \%$ cholesterol SVLS on a supported vesicle layer on a gold-coated QCM-D quartz sensor upon heating. Right panel closer view of the normalized shear viscosity first-order derivative for DMPC $+20 \%$ cholesterol SVLS. Solid lines denote multiple peak gaussian fitting.

The role of Chol in the structure and nanomechanics of SLBs has been extensively studied by AFM (L Redondo-Morata et al., 2012b; R M A Sullan et al., 2010; Al-Rekabi \& Contera, 2018; S Garcia-Manyes et al., 2010; Adhyapak et al., 2018), as well as its interplay with sphingolipids or other lipids (García-Arribas et al., 2016; 2006; Ahyayauch et al., 2002; Guyomarc'h et al., 2014) and further reviewed in (B. Gumí-Audenis et al., 2016b).

By means of temperature-controlled AFM imaging and AFM-FS to assess the influence of Chol on the membrane ordering and stability (Redondo-Morata et al., 2012), this work analyzed a DPPC:Chol in representative range of compositions up to $50 \mathrm{~mol} \% \mathrm{Chol}$, studying the phase evolution upon heating (from room temperature to temperatures high above the $T_{m}$ of DPPC) and the corresponding (nano)mechanical stability. This provided a correlation between the mechanical behavior and composition with the lateral order of each phase present in the bilayers. It proved that low Chol contents lead to a phase segregated system, while high contents of Chol can give a homogeneous topography, although a bimodal $F_{\mathrm{b}}$ distribution was observed, possibly due to the presence of small heterogeneities that could not be resolved in the images. In all cases, it was demonstrated that Chol enhances the mechanical stability of the binary 
1003

1004

1005

1006

1007

1008

1009

1010

1011

1012

1013

1014

1015

1016

1017

1018

1019

1020

1021

1022

1023

membrane, and an extraordinarily stable system was observed for equimolar fractions (50 mol $\%$ Chol). In addition, even when no thermal transition was detected by the traditional bulk analysis techniques for vesicles with high Chol content (40 and $50 \mathrm{~mol} \%$ ), it was shown that temperature-controlled AFM-FS is capable of identifying a thermal transition for the SLBs. As detailed in Figure 13, for high-Chol contents -40 and $50 \mathrm{~mol} \%$ - the DPPC:Chol system shows a double $F_{\mathrm{b}}$ distribution at room temperature, probably due to the presence of (space or time) non-resolved heterogeneities for these compositions. Although no thermal transition was observed in DSC thermograms of free-standing systems, when AFM-FS experiments were performed at different temperatures for these systems, a gradual decrease of the mean $F_{\mathrm{b}}$ value was observed until temperature reached ca. $40^{\circ} \mathrm{C}$. The continuous decrease in the $F_{\mathrm{b}}$ value with increasing temperature may be ascribed to an increase in fluidity of the system, and it is evident that, although not detected in DSC of DPPC:Chol vesicles, a transition occurs for DPPC:Chol SLBs with 40 and $50 \mathrm{~mol} \% \mathrm{Chol}$ around $42-45^{\circ} \mathrm{C}$. Below this temperature, the system in the $I_{\circ}$ phase presents extraordinary lateral order and may have properties that resemble a gel-like bilayer, and as temperature increases, it gradually becomes a system with higher lateral mobility (but still with higher order than the DPPC / phase). This behavior indicates that for DPPC:Chol SLBs, a thermal transition occurs even for high-Chol compositions, in a temperature range between ca. $42-47^{\circ} \mathrm{C}$ independently of the membrane composition, and that it may probably be of second-order for the high-Chol DPPC:Chol bilayers (40-50 mol \%), which generally appear as a very broad transition or it is even not detected in DSC experiments of free standing vesicles or QCM-D of SVLS. 

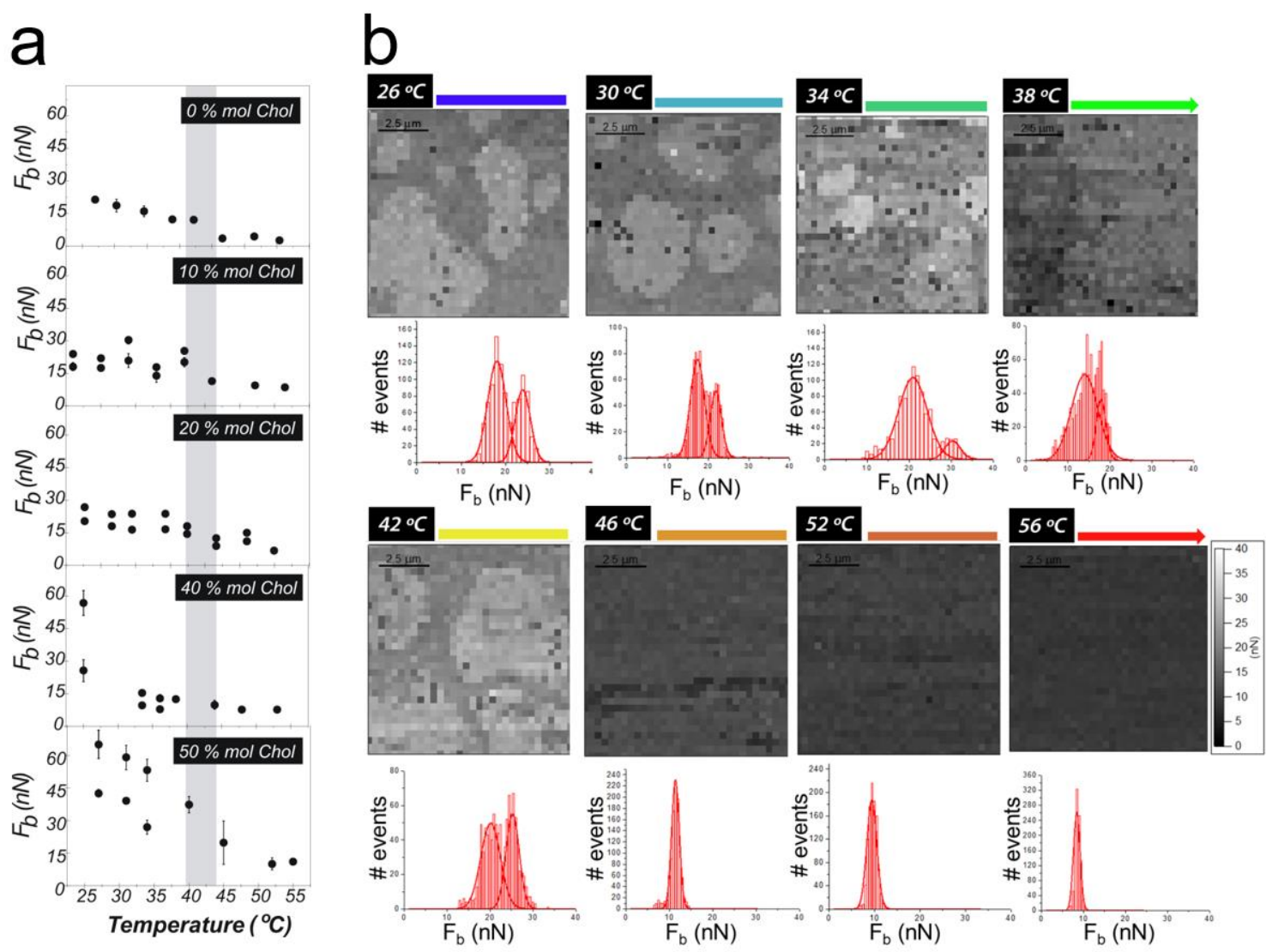

1024

1025

1026

1027

1028

1029

1030

1031

1032

1033

1034

1035

1036

1037

1038

1039

1040

1041

1042

Figure 13. a) Mean $F_{b}$ value of DPPC:Chol SLBs in $10 \mathrm{mM} \mathrm{HEPES,} 20 \mathrm{mM} \mathrm{MgCl}_{2}$ and $150 \mathrm{mM} \mathrm{NaCl}$, $\mathrm{pH}$ 7.4, with various Chol contents, as a function of temperature. The shadowed vertical line marks the temperature range where the main transition in pure DPPC occurs. For DPPC:Chol SLBs with 40 and $50 \mathrm{~mol} \% \mathrm{Chol}$, although not detected in DSC of DPPC:Chol vesicles, a transition occurs around $42-45^{\circ} \mathrm{C}$. b) $\mathrm{F}_{\mathrm{b}}$ maps and distributions for DPPC:Chol SPB with $10 \mathrm{~mol} \% \mathrm{Chol}$, in $10 \mathrm{mM}$ HEPES, $20 \mathrm{mM} \mathrm{MgCl}_{2}$ and $150 \mathrm{mM} \mathrm{NaCl}, \mathrm{pH}$ 7.4, with increasing temperature. Reprinted from (L Redondo-Morata et al., 2012b).

Strikingly, information about the lateral in-plane structure of such systems can be instead obtained by grazing incidence XR diffraction (GIXD). In (Gumi-Audenis et al., 2018), a silicon-SLB system was designed in order to deal with the requirement of the wetting preservation to guarantee the membrane stability while minimizing the background from the liquid environment. Using this methodology, it was confirmed that DPPC:Chol with low contents of Chol up to $30 \mathrm{~mol} \%$ lead to separation into two coexisting phases at room temperature and concentrations higher than $30 \mathrm{~mol} \%$ Chol appear to be a unique $I_{0}$ phase. This condition was consistent with the earlier work (Redondo-Morata et al., 2012b) demonstrating that such increase of nanomechanical resistance is associated to a strong lateral interaction mediated by Chol molecules placed between the DPPC ones, due to a highly stable structure with most probably an equimolar DPPC:Chol ratio. 
1043

1044

1045

1046

1047

1048

1049

1050

1051

1052

1053

1054

1055

1056

1057

1058

1059

1060

1061

1062

1063

1064

1065

1066

1067

1068

1069

1070

1071

1072

1073

1074

1075

To date, efforts continue to understand the complex role of cholesterol in biomembranes. Still, these discoveries cannot always be directly translated to the behavior of living cell membranes. By means of advanced fluorescence imaging and spectroscopy, Sezgin and colleagues (Pinkwart et al., 2019) have shown that cholesterol diffuses faster than phospholipids in live membranes, but not in model membranes. Interestingly, a detailed statistical diffusion analysis suggested two-component diffusion for cholesterol in the plasma membrane of live cells.

Recent work based on a systematic AFM-FS study on DOPC:Chol SLBs at varying ratios suggested, however, that cholesterol may not just be responsible of increasing the mechanical stability but also introduce irregularities across the leaflets (Adhyapak et al., 2018). At Chol ratios above 20\%, they could observe two sudden jumps in the force-distance curve, the second jump always displaying shorten separation. They interpreted that cholesterol induced asymmetry across the (in the inner and outer leaflets) bilayer, related to the phenomena of interleaflet coupling and depending on Chol concentration.

Recently, Contera and coworkers took a different approach to study the effect of Chol in the mechanical properties of lipid bilayers (Al-Rekabi \& Contera, 2018). Applying different frequencies simultaneously in an AFM topography mode, namely amplitude modulationfrequency modulation (AM-FM), they investigated the effect of Chol on DPPC bilayers in concentrations from 0 to $60 \%$. The lipid bilayers probed with this methodology displayed both elastic and viscous behavior. Cholesterol showed to modulates both, increasing elastic and viscous components as a function of Chol concentrations. The authors interpreted that their results may have a physiological relevance, where such viscoelastic properties may be used by cells to modulate the propagation (elastic) or attenuation (viscous) of mechanical signals across the cell membrane.

\section{Connection between nanoscale measurements and} thermodynamic descriptors of membranes

When studying phase coexistence in lipid membranes a distinction between thermodynamically stable phases and transient local domains in lipid bilayer at equilibrium should be made. Let us briefly discuss this in line with the above-exposed results on phospholipid-cholesterol binary mixtures. PC-Chol binary mixtures have been studied for many years using a plethora of experimental techniques. Yet, despite their apparent simplicity being two-component systems, their phase behavior remains controversial and, in many cases, departs from the canonical phase diagram proposed by Vist and Davis (Vist \& Davis, 1990). In fact, results depend strongly on the 
1076

1077

1078

1079

1080

1081

1082

1083

1084

1085

1086

1087

1088

1089

1090

1091

1092

1093

1094

1095

1096

1097

1098

1099

1100

1101

1102

1103

1104

1105

1106

1107

1108

spatio-temporal resolution of the different experimental techniques. For instance, unlike predicted by most phase diagrams, $I_{\mathrm{d}}-I_{0}$ phase coexistence was not observed for micron-sized GUVs of DPPC-cholesterol mixtures using fluorescence microscopy (Veatch \& Keller, 2003), while some other techniques captured this phenomenon for free standing MLVs vesicles (DSC (Mannock et al., 2010), volumetric (Miyoshi et al., 2014), NMR (Davis et al., 2009), SLBs (AFM)(L Redondo-Morata et al., 2012b), and SVLs (QCM-D)(Losada-Pérez, Khorshid, et al., 2015). These findings suggest that, instead of macroscopic phase separation, these systems are presumably filled with mesoscopic and nanoscopic domains, whose size and lifetime depends on temperature and mixture composition (Winkler et al., 2017).

Classically, when dealing with phase separation the Gibbs' phase rule is customarily invoked, assuming macroscopic phase coexistence and neglecting the role of phase boundaries, which comparably, hardly contribute to the free energy. If domains are small, the interfaces between domains constitute an important part of the system and its contribution to the free energy is not negligible. In this respect, using the term phase in the classical thermodynamic sense might not be appropriate (Heimburg, 1998; Almeida et al., 2005). Following Hill (Hill, 2013) a system can no longer be called a phase when it ceases to behave like a macroscopic system and starts behaving like a small system.

In this respect, both AFM and QCM-D have demonstrated to be valuable tools to detect domain (nano/mesoscopic sized domains) coexistence within supported membranes of binary lipid mixtures.

\section{Conclusions and future perspectives}

As mentioned in the previous sections, AFM-FS can interestingly resolve coexistence of domains at concentrations where height differences at domain boundaries are not detectable. Breakthrough force thus stands as a useful mechanical descriptor to resolve domain coexistence. Even though domains cannot be considered as phases in the purely thermodynamic standpoint, this does not mean that these systems cannot be treated in a rigorous thermodynamic way. As a matter of fact, the breakthrough force is able to detect a thermal transition at high cholesterol concentrations in binary PC:Chol SLBs, whose origin deserves to be revisited and further explored in the framework of either fluctuations arising in the vicinity of a critical point, the possibility of glassy-cholesterol-rich nanodomains or eventual cholesterol crystallization. In this sense, the development of HS-AFM will likely provide new insights due to its unprecedented spatio-temporal resolution, thanks to a direct correlation between structure and diffusion (Munguira et al., 2016). Similarly, HS Force Spectroscopy has the capacity to cover a wider force 
1109

1110

1111

1112

1113

1114

1115

1116

1117

1118

1119

1120

1121

1122

1123

1124

1125

1126

1127

1128

1129

1130

1131

1132

1133

1134

1135

1136

1137

1138

1139

spectrum, which can help to validate theoretical frameworks of the field, as well as provide a direct correlation with Molecular Dynamics simulations (Rico et al., 2013).

There is indeed plenty of room in fundamental lipid biophysics and phase behavior in particular to use both purely mechanical and viscoelastic properties as descriptors to provide a more complete picture of the origins of domain formation and coexistence. The ultimate lipid phase coexistence to be fully understood are transient nanodomains, often (confusively) referred to as lipid rafts. There is to date no agreement about the size and lifetime of rafts, and the term is used to referred to a collection of different structures (Goñi, 2019). Based on the current knowledge, microdomains in equilibrium are no longer considered suitable models for the biological structure that rafts represent. Fortunately, advanced super-resolution microscopies and fast techniques are becoming available and will shed some light in one of the most discussed topics in biomembrane studies.

In light of this, multiscale spatio-temporal measurements of mechanical properties of supported membranes can help to experimentally address different scenarios where membrane micro- and nano-domain formation finds theoretical support. Starting from equilibrium ternary lipid mixtures in the vicinity of a critical point, it might be worth exploring how the presence of critical fluctuations reflects in the membrane mechanical properties, and if these properties can be used as physical descriptors to test critical-point universality laws. Secondly, tuning membrane (local and global) environment by actively controlling curvature, leaflet asymmetry, solid support topography and addition of external components would help complete the manifold where domain formation occurs. Finally, the growing spatiotemporal capabilities of AFM are promising tools to explore membrane organization in the realm of non-equilibrium phenomena.

\section{Acknowledgements}

We acknowledge financial support from the Generalitat de Catalunya (2017-SGR-1442), the Instituto de Salud Carlos III, through "Acciones CIBER" and CIBER-BBN FlexQS-skin and FelxCAB projects. This work was also supported by a grant overseen by the French National Research Agency (ANR) as part of the "Investments d'Avenir" Programme (I-SITE ULNE / ANR-16-IDEX0004 ULNE) to L.R.-M. Financial support from the project 'SADI' by the programme Action Recherche Concertée, Université Libre de Bruxelles (ULB) to P.L-P is also acknowledged.

\section{Glossary of lipid acronyms}


C16:0 palmitoyl Cer (aka palmitoyl Cer, $p$ Cer)

C16:0 palmitoyl SM laka palmitoyl SM)

C24:1 nervonoyl Cer laka nervonoyl Cer, $n$ Cer)

C24:1 nervonoyl SM (aka nervonoyl $S M$ )

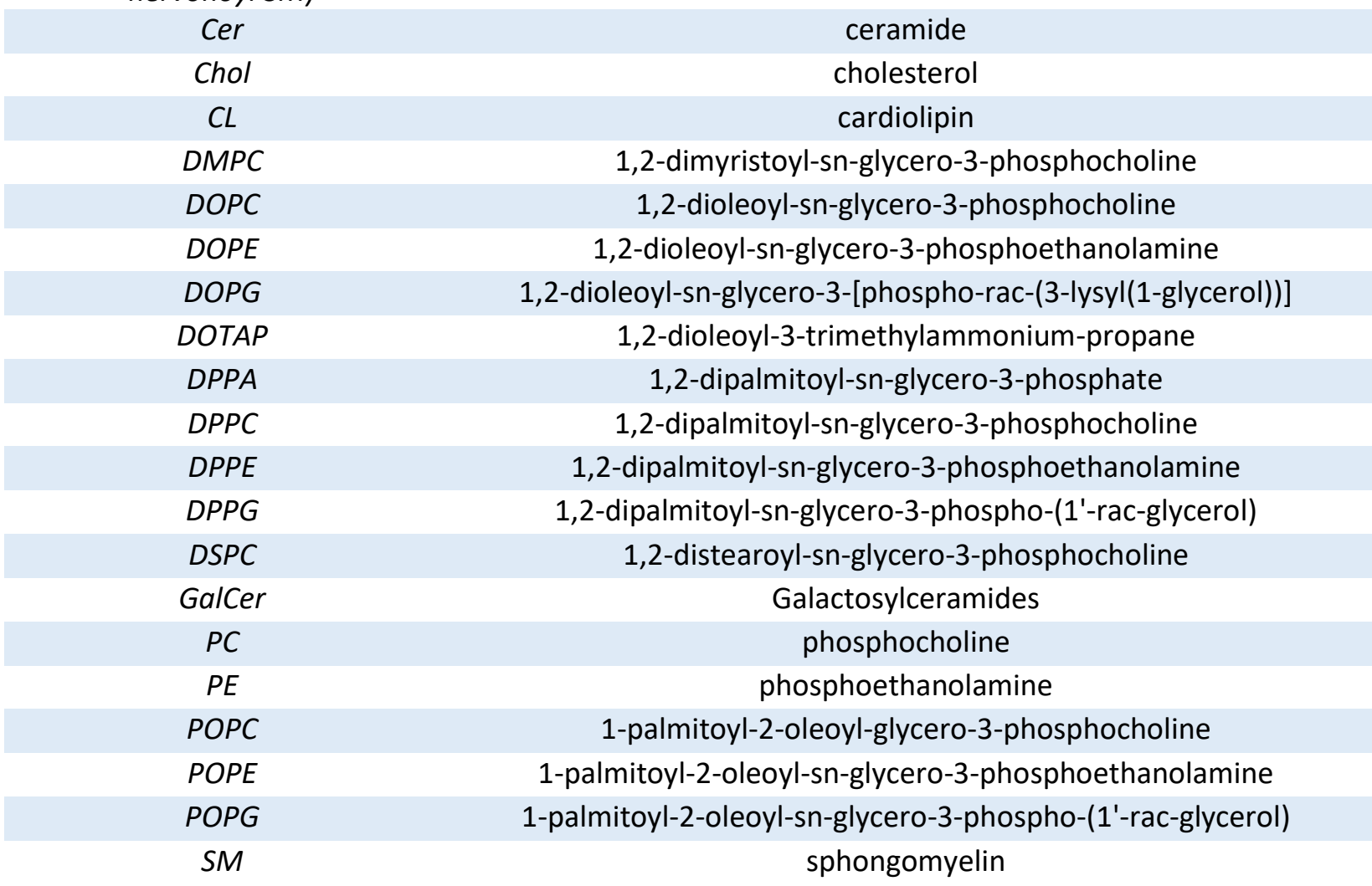

N-palmitoyl-D-erythro-sphingosine

$\mathrm{N}$-palmitoyl-D-erythro-sphingosylphosphorylcholine

$\mathrm{N}$-nervonoyl-D-erythro-sphingosine

N-Nervonoyl-D-erythro-sphingosylphosphorylcholine-d9

$$
\begin{gathered}
\text { ceramide } \\
\text { cholesterol }
\end{gathered}
$$

1,2-dimyristoyl-sn-glycero-3-phosphocholine

1,2-dioleoyl-sn-glycero-3-phosphocholine

oleoyl-sn-glycero-3-[phospho-rac-(3-lysyl(1-glycerol))]

1,2-dipalmitoyl-sn-glycero-3-phosphate

,2-dipalmitoyl-sn-glycero-3-phosphoethanolamine

1,2-dipalmitoyl-sn-glycero-3-phospho-(1'-rac-glycerol)

Galactosylceramides

phosphocholine

phosphoethanolamine

\section{References}

Adhyapak, P. R., Panchal, S. V., \& Murthy, A. V. R. (2018). Cholesterol induced asymmetry in DOPC bilayers probed by AFM force spectroscopy. Biochimica et Biophysica Acta (BBA) - Biomembranes, 1860(5), 953-959. https://doi.org/10.1016/j.bbamem.2018.01.021

Ahyayauch, H., Requero, M. A., Alonso, A., Bennouna, M., \& Goni, F. M. (2002). Surfactant effects of chlorpromazine and imipramine on lipid bilayers containing sphingomyelin and cholesterol. Journal of Colloid and Interface Science, 256(2), 284-289. https://doi.org/10.1006/jcis.2002.8690 
Alessandrini, A., \& Facci, P. (2012). Nanoscale mechanical properties of lipid bilayers and their relevance in biomembrane organization and function. Micron, 43(12), 1212-1223. https://doi.org/10.1016/j.micron.2012.03.013

Almeida, P. F. F., Pokorny, A., \& Hinderliter, A. (2005). Thermodynamics of membrane domains. Biochimica et Biophysica Acta (BBA) - Biomembranes, 1720(1), 1-13. https://doi.org/10.1016/j.bbamem.2005.12.004

Al-Rekabi, Z., \& Contera, S. (2018). Multifrequency AFM reveals lipid membrane mechanical properties and the effect of cholesterol in modulating viscoelasticity. Proceedings of the National Academy of Sciences, 115(11), 2658-2663. https://doi.org/10.1073/pnas.1719065115

Ando, T, Uchihashi, T., Kodera, N., Yamamoto, D., Miyagi, A., Taniguchi, M., \& Yamashita, H. (2008). High-speed AFM and nano-visualization of biomolecular processes. Pflugers Archiv-European Journal of Physiology, 456(1), 211-225. https://doi.org/10.1007/s00424-007-0406-0

Ando, Toshio. (2017). High-speed atomic force microscopy and its future prospects. Biophysical Reviews, 10(2), 285-292. https://doi.org/10.1007/s12551-017-0356-5

Armond, J. W., Macpherson, J. V, \& Turner, M. S. (2011). Pulling Nanotubes from Supported Bilayers. Langmuir, 27(13), 8269-8274.

Bangham, A. D., \& Horne, R. W. (1964). Negative staining of phospholipids and their structural modification by surface-active agents as observed in the electron microscope. Journal of Molecular Biology, 8(5), 660-IN10. https://doi.org/10.1016/S0022-2836(64)80115-7

BANGHAM, A. D., PETHICA, B. A., \& SEAMAN, G. V. (1958). The charged groups at the interface of some blood cells. The Biochemical Journal, 69(1), 12-19. https://doi.org/10.1042/bj0690012 
Barrett, R. C. (1991). High-speed, large-scale imaging with the atomic force microscope. Journal of Vacuum Science \& Technology B: Microelectronics and Nanometer Structures, 9(2), 302. https://doi.org/10.1116/1.585610

Berg, J. M., Tymoczko, J. L., Stryer, L., Berg, J. M., Tymoczko, J. L., \& Stryer, L. (2002). Biochemistry (5th ed.). W H Freeman.

Bernchou, U., Ipsen, J. H., \& Simonsen, A. C. (2009). Growth of solid domains in model membranes: Quantitative image analysis reveals a strong correlation between domain shape and spatial position. The Journal of Physical Chemistry. B, 113(20), 7170-7177. https://doi.org/10.1021/jp809989t

Binnig, G., Quate, C. F., \& Gerber, C. (1986). ATOMIC FORCE MICROSCOPE. Physical Review Letters, 56(9), 930-933. https://doi.org/10.1103/PhysRevLett.56.930

Blanchette, C. D., Lin, W.-C., Ratto, T. V., \& Longo, M. L. (2006). Galactosylceramide Domain Microstructure: Impact of Cholesterol and Nucleation/Growth Conditions. Biophysical Journal, 90(12), 4466-4478. https://doi.org/10.1529/biophysj.105.072744

Brochard-Wyart, F., Borghi, N., Cuvelier, D., \& Nassoy, P. (2006). Hydrodynamic narrowing of tubes extruded from cells. Proceedings of the National Academy of Sciences, 103(20), 7660.

Butt, H. J., \& Franz, V. (2002). Rupture of molecular thin films observed in atomic force microscopy. I. Theory. Physical Review E, 66(3). https://doi.org/10.1103/PhysRevE.66.031601

Canham, P. B. (1970). The minimum energy of bending as a possible explanation of the biconcave shape of the human red blood cell. Journal of Theoretical Biology, 26(1), 6181.

Chiantia, S, Kahya, N., Ries, J., \& Schwille, P. (2006). Effects of ceramide on liquid-ordered domains investigated by simultaneous AFM and FCS. Biophysical Journal, 90(12), 4500-4508. https://doi.org/10.1529/biophysj.106.081026 
Chiantia, S, Ries, J., Kahya, N., \& Schwille, P. (2006). Combined AFM and two-focus SFCS study of raft-exhibiting model membranes. Chemphyschem, 7(11), 2409-2418. https://doi.org/10.1002/cphc.200600464

Chiantia, Salvatore, Kahya, N., \& Schwille, P. (2007). Raft domain reorganization driven by short- and long-chain ceramide: A combined AFM and FCS study. Langmuir, 23(14), 7659-7665. https://doi.org/10.1021/la7010919

Chiantia, Salvatore, Ries, J., Chwastek, G., Carrer, D., Li, Z., Bittman, R., \& Schwille, P. (2008). Role of ceramide in membrane protein organization investigated by combined AFM and FCS. Biochimica Et Biophysica Acta-Biomembranes, 1778(5), 1356-1364. https://doi.org/10.1016/j.bbamem.2008.02.008

Chiaruttini, N., Redondo-Morata, L., Colom, A., Humbert, F., Lenz, M., Scheuring, S., \& Roux, A. (2015). Relaxation of Loaded ESCRT-III Spiral Springs Drives Membrane Deformation. Cell, 163(4), 866-879. https://doi.org/10.1016/j.cell.2015.10.017

Cho, N.-J., Frank, C. W., Kasemo, B., \& Höök, F. (2010). Quartz crystal microbalance with dissipation monitoring of supported lipid bilayers on various substrates. Nature Protocols, 5(6), 1096-1106. https://doi.org/10.1038/nprot.2010.65

Cho, N.-J., Kanazawa, K. K., Glenn, J. S., \& Frank, C. W. (2007). Employing Two Different Quartz Crystal Microbalance Models To Study Changes in Viscoelastic Behavior upon Transformation of Lipid Vesicles to a Bilayer on a Gold Surface. Analytical Chemistry, 79(18), 7027-7035. https://doi.org/10.1021/ac0709504

Choi, S.-E., Greben, K., Wördenweber, R., \& Offenhäusser, A. (2016). Positively charged supported lipid bilayer formation on gold surfaces for neuronal cell culture. Biointerphases, 11(2), 21003. https://doi.org/10.1116/1.4945306

Clausen-Schaumann, H., Rief, M., Tolksdorf, C., \& Gaub, H. E. (2000). Mechanical stability of single DNA molecules. Biophysical Journal, 78(4), 1997-2007. 
Crespo-Villanueva, A., Gumí-Audenis, B., Sanz, F., Artzner, F., Mériadec, C., Rousseau, F., Lopez, C., Giannotti, M. I., \& Guyomarc'h, F. (2018). Casein interaction with lipid membranes: Are the phase state or charge density of the phospholipids affecting protein adsorption? Biochimica Et Biophysica Acta. Biomembranes, 1860(12), 2588-2598. https://doi.org/10.1016/j.bbamem.2018.09.016

Dai, J., \& Sheetz, M. P. (1999). Membrane Tether Formation from Blebbing Cells. Biophysical Journal, 77(6), 3363-3370.

Daillant, J., Bellet-Amalric, E., Braslau, A., Charitat, T., Fragneto, G., Graner, F., Mora, S., Rieutord, F., \& Stidder, B. (2005). Structure and fluctuations of a single floating lipid bilayer. Proceedings of the National Academy of Sciences of the United States of America, 102(33), 11639.

Davis, J. H., Clair, J. J., \& Juhasz, J. (2009). Phase Equilibria in DOPC/DPPC-d62/Cholesterol Mixtures. Biophysical Journal, 96(2), 521-539. https://doi.org/10.1016/j.bpj.2008.09.042

Deamer, D. W. (2010). From "banghasomes" to liposomes: A memoir of Alec Bangham, 19212010. The FASEB Journal : Official Publication of the Federation of American Societies for Experimental Biology, 24(5), 1308-1310. https://doi.org/10.1096/fj.10-0503

Dieluweit, S., Csiszar, A., Rubner, W., Fleischhauer, J., Houben, S., \& Merkel, R. (2010). Mechanical Properties of Bare and Protein-Coated Giant Unilamellar Phospholipid Vesicles. A Comparative Study of Micropipet Aspiration and Atomic Force Microscopy. Langmuir, 26(13), 11041-11049. https://doi.org/10.1021/la1005242

Dimova, R. (2019). Giant Vesicles and Their Use in Assays for Assessing Membrane Phase State, Curvature, Mechanics, and Electrical Properties. Annual Review of Biophysics, 48(1), 93-119. https://doi.org/10.1146/annurev-biophys-052118-115342

Doktorova, M., Heberle, F. A., Eicher, B., Standaert, R. F., Katsaras, J., London, E., Pabst, G., \& Marquardt, D. (2018). Preparation of asymmetric phospholipid vesicles for use as cell 
membrane models. Nature Protocols, 13(9), 2086-2101. https://doi.org/10.1038/s41596-018-0033-6

1252

1253

1254

1255

1256

1257

1258

1259

1260

1261

1262

1263

1264

1265

1266

1267

1268

1269

1270

1271

1272

1273

Domenech, O., Redondo, L., Picas, L., Morros, A., Montero, M. T., \& Hernandez-Borrell, J. (2007). Atomic force microscopy characterization of supported planar bilayers that mimic the mitochondrial inner membrane. Journal of Molecular Recognition, 20(6), 546-553. https://doi.org/10.1002/jmr.849

Dufrêne, Y. F., Ando, T., Garcia, R., Alsteens, D., Martinez-Martin, D., Engel, A., Gerber, C., \& Müller, D. J. (2017). Imaging modes of atomic force microscopy for application in molecular and cell biology. Nature Nanotechnology, 12, 295. https://doi.org/10.1038/nnano.2017.45

Dufrene, Y. F., Barger, W. R., Green, J. B. D., \& Lee, G. U. (1997). Nanometer-scale surface properties of mixed phospholipid monolayers and bilayers. Langmuir, 13(18), 47794784. https://doi.org/10.1021/la970221r

Dufrene, Y. F., Boland, T., Schneider, J. W., Barger, W. R., \& Lee, G. U. (1998). Characterization of the physical properties of model biomembranes at the nanometer scale with the atomic force microscope. Faraday Discussions, 111, 79-94.

Dufrêne, Y. F., \& Lee, G. U. (2000). Advances in the characterization of supported lipid films with the atomic force microscope. Biochimica et Biophysica Acta (BBA) Biomembranes, 1509(1), 14-41. https://doi.org/10.1016/\$0005-2736(00)00346-1

Ehrig, J., Petrov, E. P., \& Schwille, P. (2011). Phase separation and near-critical fluctuations in two-component lipid membranes: Monte Carlo simulations on experimentally relevant scales. New Journal of Physics, 13(4), 045019. https://doi.org/10.1088/1367$2630 / 13 / 4 / 045019$

El Kirat, K., Morandat, S., \& Dufrene, Y. F. (2010). Nanoscale analysis of supported lipid bilayers using atomic force microscopy. Biochimica Et Biophysica Acta-Biomembranes, 1798(4), 750-765. https://doi.org/10.1016/j.bbamem.2009.07.026 
Evans, E, \& Ritchie, K. (1997). Dynamic strength of molecular adhesion bonds. Biophysical Journal, 72(4), 1541-1555.

Evans, E, \& Yeung, A. (1994). Hidden dynamics in rapid changes of bilayer shape. Chemistry and Physics of Lipids, 73(1), 39-56. https://doi.org/10.1016/0009-3084(94)90173-2

Evans, Evan, Heinrich, V., Ludwig, F., \& Rawicz, W. (2003). Dynamic Tension Spectroscopy and Strength of Biomembranes. Biophysical Journal, 85(4), 2342-2350.

Fidorra, M., Heimburg, T., \& Seeger, H. M. (2009). Melting of individual lipid components in binary lipid mixtures studied by FTIR spectroscopy, DSC and Monte Carlo simulations. Biochimica Et Biophysica Acta-Biomembranes, 1788(3), 600-607. https://doi.org/10.1016/j.bbamem.2008.12.003

Florin, E. L., Moy, V. T., \& Gaub, H. E. (1994). Adhesion forces between individual ligandreceptor pairs. Science, 264(5157), 415 LP - 417. https://doi.org/10.1126/science. 8153628

Franz, V., Loi, S., Muller, H., Bamberg, E., \& Butt, H. H. (2002). Tip penetration through lipid bilayers in atomic force microscopy. Colloids and Surfaces B-Biointerfaces, 23(2-3), 191-200.

García-Arribas, A. B., Alonso, A., \& Goñi, F. M. (2016). Cholesterol interactions with ceramide and sphingomyelin. Chemistry and Physics of Lipids, 199, 26-34. https://doi.org/10.1016/j.chemphyslip.2016.04.002

García-Arribas, A. B., González-Ramírez, E. J., Sot, J., Areso, I., Alonso, A., \& Goñi, F. M. (2017). Complex Effects of 24:1 Sphingolipids in Membranes Containing Dioleoylphosphatidylcholine and Cholesterol. Langmuir, 33(22), 5545-5554. https://doi.org/10.1021/acs.langmuir.7b00162

Garcia-Manyes, S, Guell, A. G., Gorostiza, P., \& Sanz, F. (2005). Nanomechanics of silicon surfaces with atomic force microscopy: An insight to the first stages of plastic deformation. Journal of Chemical Physics, 123(11). https://doi.org/10.1063/1.2035094 
Garcia-Manyes, S, Oncins, G., \& Sanz, F. (2005a). Effect of ion-binding and chemical phospholipid structure on the nanomechanics of lipid bilayers studied by force spectroscopy. Biophysical Journal, 89, 1812-1826. https://doi.org/10.1529/biophysj.105.064030

Garcia-Manyes, S, Oncins, G., \& Sanz, F. (2005b). Effect of temperature on the nanomechanics of lipid bilayers studied by force spectroscopy. Biophysical Journal, 89(6), 4261-4274. https://doi.org/10.1529/biophysj.105.065581

Garcia-Manyes, S, Redondo-Morata, L., Oncins, G., \& Sanz, F. (2010). Nanomechanics of Lipid Bilayers: Heads or Tails? Journal of the American Chemical Society, 132(37), 1287412886. https://doi.org/10.1021/ja1002185

Garcia-Manyes, S, \& Sanz, F. (2010). Nanomechanics of lipid bilayers by force spectroscopy with AFM: A perspective. Biochimica Et Biophysica Acta-Biomembranes, 1798(4), 741749. https://doi.org/10.1016/j.bbamem.2009.12.019

Garcia-Manyes, Sergi, Redondo-Morata, L., Oncins, G., \& Sanz, F. (2010). Nanomechanics of Lipid Bilayers: Heads or Tails? Journal of the American Chemical Society, 132(37), 12874-12886. https://doi.org/10.1021/ja1002185

Giannotti, M. I., \& Vancso, G. J. (2007). Interrogation of single synthetic polymer chains and polysaccharides by AFM-based force spectroscopy. ChemPhysChem, 8(16). https://doi.org/10.1002/cphc.200700175

Giles, R., Cleveland, J. P., Manne, S., Hansma, P. K., Drake, B., Maivald, P., Boles, C., Gurley, J., \& Elings, V. (1993). Noncontact force microscopy in liquids. Applied Physics Letters, 63(5), 617-618. https://doi.org/10.1063/1.109967

Gillissen, J. J. J., Jackman, J. A., Tabaei, S. R., Yoon, B. K., \& Cho, N.-J. (2017). Quartz Crystal Microbalance Model for Quantitatively Probing the Deformation of Adsorbed Particles at Low Surface Coverage. Analytical Chemistry, 89(21), 11711-11718. https://doi.org/10.1021/acs.analchem.7b03179 
Goñi, F. M. (2019a). "Rafts": A nickname for putative transient nanodomains. Chemistry and Physics of Lipids, 218, 34-39. https://doi.org/10.1016/j.chemphyslip.2018.11.006

Goñi, F. M. (2019b). "Rafts": A nickname for putative transient nanodomains. In Chemistry and Physics of Lipids (Vol. 218, pp. 34-39). Elsevier Ireland Ltd. https://doi.org/10.1016/j.chemphyslip.2018.11.006

González-Ramírez, E. J., Artetxe, I., García-Arribas, A. B., Goñi, F. M., \& Alonso, A. (2019). Homogeneous and Heterogeneous Bilayers of Ternary Lipid Compositions Containing Equimolar Ceramide and Cholesterol. Langmuir, 35(15), 5305-5315. https://doi.org/10.1021/acs.langmuir.9b00324

Gumí-Audenis, B., Costa, L., Carlá, F., Comin, F., Sanz, F., \& Giannotti, M. I. (2016a). Structure and nanomechanics of model membranes by atomic force microscopy and spectroscopy: Insights into the role of cholesterol and sphingolipids. Membranes, 6(4). https://doi.org/10.3390/membranes6040058

Gumí-Audenis, B., Costa, L., Carlá, F., Comin, F., Sanz, F., \& Giannotti, M. I. (2016b). Structure and nanomechanics of model membranes by atomic force microscopy and spectroscopy: Insights into the role of cholesterol and sphingolipids. Membranes, 6(4). https://doi.org/10.3390/membranes6040058

Gumí-Audenis, B., Costa, L., Redondo-Morata, L., Milhiet, P.-E., Sanz, F., Felici, R., Giannotti, M. I., \& Carlà, F. (2018). In-plane molecular organization of hydrated single lipid bilayers: DPPC: cholesterol. Nanoscale, 10(1). https://doi.org/10.1039/c7nr07510c

Gumi-Audenis, B., Costa, L., Redondo-Morata, L., Milhiet, P.-E., Sanz, F., Felici, R., Giannotti, M. I., \& Carla, F. (2018). In-plane molecular organization of hydrated single lipid bilayers: DPPC:cholesterol. Nanoscale, 10(1), 87-92. https://doi.org/10.1039/c7nr07510c

Gumí-Audenis, Berta, Costa, L., Ferrer-Tasies, L., Ratera, I., Ventosa, N., Sanz, F., \& Giannotti, M. I. (2018). Pulling lipid tubes from supported bilayers unveils the underlying 
substrate contribution to the membrane mechanics. Nanoscale. https://doi.org/10.1039/C8NR03249A

Gumí-Audenis, Berta, Sanz, F., \& Giannotti, M. I. (2015). Impact of galactosylceramides on the nanomechanical properties of lipid bilayer models: An AFM-force spectroscopy study. Soft Matter, 11(27), 5447-5454. https://doi.org/10.1039/C5SM01252」

Guyomarc'h, F., Zou, S., Chen, M., Milhiet, P.-E., Godefroy, C., Vié, V., \& Lopez, C. (2014). Milk Sphingomyelin Domains in Biomimetic Membranes and the Role of Cholesterol: Morphology and Nanomechanical Properties Investigated Using AFM and Force Spectroscopy. Langmuir, 30(22), 6516-6524. https://doi.org/10.1021/la501640y

Hardy, G. J., Nayak, R., \& Zauscher, S. (2013). Model cell membranes: Techniques to form complex biomimetic supported lipid bilayers via vesicle fusion. Current Opinion in Colloid \& Interface Science, 18(5), 448-458. https://doi.org/10.1016/j.cocis.2013.06.004

Hasan, I. Y., \& Mechler, A. (2015). Viscoelastic changes measured in partially suspended single bilayer membranes. Soft Matter, 11(27), 5571-5579. https://doi.org/10.1039/C5SM00278H

Hassinger, J. E., Oster, G., Drubin, D. G., \& Rangamani, P. (2017). Design principles for robust vesiculation in clathrin-mediated endocytosis. Proceedings of the National Academy of Sciences, 114(7), E1118 LP-E1127. https://doi.org/10.1073/pnas.1617705114

Heimburg, T. (1998). Mechanical aspects of membrane thermodynamics. Estimation of the mechanical properties of lipid membranes close to the chain melting transition from calorimetry. Biochimica Et Biophysica Acta-Biomembranes, 1415(1), 147-162. https://doi.org/10.1016/s0005-2736(98)00189-8

Hill, T. L. (2013). Thermodynamics of Small Systems/ (Reprint). Dover Publications Inc. 
Hochmuth, F. M., Shao, J. Y., Dai, J., \& Sheetz, M. P. (1996). Deformation and flow of membrane into tethers extracted from neuronal growth cones. Biophysical Journal, 70(1), 358-369.

Hohner, A. O., David, M. P. C., \& Rädler, J. O. (2010). Controlled solvent-exchange deposition of phospholipid membranes onto solid surfaces. Biointerphases, 5(1), 1-8. https://doi.org/10.1116/1.3319326

Hugel, T., \& Seitz, M. (2001). The Study of Molecular Interactions by AFM Force Spectroscopy. Macromolecular Rapid Communications, 22(13), 989-1016. https://doi.org/10.1002/1521-3927(20010901)22:13<989::AID-MARC989>3.0.CO;2-D

Hung, W.-C., Lee, M.-T., Chen, F.-Y., \& Huang, H. W. (2007). The condensing effect of cholesterol in lipid bilayers. Biophysical Journal, 92(11), 3960-3967. https://doi.org/10.1529/biophysj.106.099234

Hurley, J. H., Boura, E., Carlson, L.-A., \& Rózycki, B. (2010). Membrane Budding. Cell, 143(6), 875-887. https://doi.org/10.1016/j.cell.2010.11.030

Ira, Zou, S., Ramirez, D. M. C., Vanderlip, S., Ogilvie, W., Jakubek, Z. J., \& Johnston, L. J. (2009). Enzymatic generation of ceramide induces membrane restructuring: Correlated AFM and fluorescence imaging of supported bilayers. Journal of Structural Biology, 168(1), 78-89. https://doi.org/10.1016/j.jsb.2009.03.014

Jiménez-Rojo, N., García-Arribas, A. B., Sot, J., Alonso, A., \& Goñi, F. M. (2014). Lipid bilayers containing sphingomyelins and ceramides of varying $\mathrm{N}$-acyl lengths: A glimpse into sphingolipid complexity. Biochimica et Biophysica Acta-Biomembranes, 1838(1 PARTB), 456-464. https://doi.org/10.1016/j.bbamem.2013.10.010

Jing, Y., Trefna, H., Persson, M., Kasemo, B., \& Svedhem, S. (2013). Formation of supported lipid bilayers on silica: Relation to lipid phase transition temperature and liposome size. Soft Matter, 10(1), 187-195. https://doi.org/10.1039/C3SM50947H 
Johnston, L. J. (2007). Nanoscale imaging of domains in supported lipid membranes. Langmuir: The ACS Journal of Surfaces and Colloids, 23(11), 5886-5895. https://doi.org/10.1021/la070108t

Kahya, N., Scherfeld, D., Bacia, K., \& Schwille, P. (2004). Lipid domain formation and dynamics in giant unilamellar vesicles explored by fluorescence correlation spectroscopy. Journal of Structural Biology, 147(1), 77-89. https://doi.org/10.1016/j.jsb.2003.09.021

Kechagia, J. Z., Ivaska, J., \& Roca-Cusachs, P. (2019). Integrins as biomechanical sensors of the microenvironment. Nature Reviews Molecular Cell Biology, 20(8), 457-473. https://doi.org/10.1038/s41580-019-0134-2

Keller, C. A., \& Kasemo, B. (1998). Surface specific kinetics of lipid vesicle adsorption measured with a quartz crystal microbalance. Biophysical Journal, 75(3), 1397-1402.

Keller, D., Larsen, N. B., Moller, I. M., \& Mouritsen, O. G. (2005). Decoupled phase transitions and grain-boundary melting in supported phospholipid bilayers. Physical Review Letters, 94(2). https://doi.org/10.1103/PhysRevLett.94.025701

Kodera, N., Yamamoto, D., Ishikawa, R., \& Ando, T. (2010). Video imaging of walking myosin V by high-speed atomic force microscopy. Nature, 468(7320). https://doi.org/10.1038/nature09450

Kurniawan, J., Ventrici de Souza, J. F., Dang, A. T., Liu, G., \& Kuhl, T. L. (2018). Preparation and Characterization of Solid-Supported Lipid Bilayers Formed by Langmuir-Blodgett Deposition: A Tutorial. Langmuir, 34(51), 15622-15639. https://doi.org/10.1021/acs.langmuir.8b03504

Lee, G. U., Chrisey, L. A., \& Colton, R. J. (1994). DIRECT MEASUREMENT OF THE FORCES BETWEEN COMPLEMENTARY STRANDS OF DNA. Science, 266(5186), 771-773. https://doi.org/10.1126/science.7973628

Leonenko, Z. V, Finot, E., Ma, H., Dahms, T. E. S., \& Cramb, D. T. (2004). Investigation of temperature-induced phase transitions in DOPC and DPPC phospholipid bilayers using 
temperature-controlled scanning force microscopy. Biophysical Journal, 86(6), 37833793. https://doi.org/10.1529/biophysj.103.036681

Leonenko, Z. V., Finot, E., Ma, H., Dahms, T. E. S., \& Cramb, D. T. (2004). Investigation of 3793. https://doi.org/10.1529/biophysj.103.036681

Lin, D. C., Dimitriadis, E. K., \& Horkay, F. (2007). Robust strategies for automated AFM force curve analysis-I. Non-adhesive indentation of soft, inhomogeneous materials. Journal of Biomechanical Engineering, 129(3), 430-440. https://doi.org/10.1115/1.2720924

Lin, W.-C., Blanchette, C. D., \& Longo, M. L. (2007). Fluid-Phase Chain Unsaturation Controlling Domain Microstructure and Phase in Ternary Lipid Bilayers Containing GalCer and Cholesterol. Biophysical Journal, 92(8), 2831-2841. https://doi.org/10.1529/biophysj.106.095422

Losada-Pérez, P., Jiménez-Monroy, K. L., Grinsven, B. van, Leys, J., Janssens, S. D., Peeters, M., 
transitions in lipid vesicles detected by a complementary set of methods: Heat-transfer measurements, adiabatic scanning calorimetry, and dissipation-mode quartz crystal microbalance. Physica Status Solidi (a), 211(6), 1377-1388. https://doi.org/10.1002/pssa.201431060

Losada-Pérez, P., Khorshid, M., Yongabi, D., \& Wagner, P. (2015). Effect of Cholesterol on the Phase Behavior of Solid-Supported Lipid Vesicle Layers. The Journal of Physical Chemistry B, 119(15), 4985-4992. https://doi.org/10.1021/acs.jpcb.5b00712

Losada-Pérez, P., Mertens, N., de Medio-Vasconcelos, B., Slenders, E., Leys, J., Peeters, M., van Grinsven, B., Gruber, J., Glorieux, C., Pfeiffer, H., Wagner, P., \& Thoen, J. (2015). Phase Transitions of Binary Lipid Mixtures: A Combined Study by Adiabatic Scanning Calorimetry and Quartz Crystal Microbalance with Dissipation Monitoring [Research Article]. Advances in Condensed Matter Physics; Hindawi. https://doi.org/10.1155/2015/479318

Mabrey, S., \& Sturtevant, J. M. (1976). Investigation of phase transitions of lipids and lipid mixtures by high sensitivity differential scanning calorimetry. Proceedings of the National Academy of Sciences of the United States of America, 73(11), 3862-3866. https://doi.org/10.1073/pnas.73.11.3862

Maeda, N., Senden, T. J., \& di Meglio, J.-M. (2002). Micromanipulation of phospholipid bilayers by atomic force microscopy. Biochimica et Biophysica Acta (BBA) - Biomembranes, 1564(1), 165-172.

Mannock, D. A., Lewis, R. N. A. H., \& McElhaney, R. N. (2010). A calorimetric and spectroscopic comparison of the effects of ergosterol and cholesterol on the thermotropic phase behavior and organization of dipalmitoylphosphatidylcholine bilayer membranes. Biochimica Et Biophysica Acta, 1798(3), 376-388. https://doi.org/10.1016/j.bbamem.2009.09.002 
Marcus, W. D., \& Hochmuth, R. M. (2002). Experimental Studies of Membrane Tethers Formed from Human Neutrophils. Annals of Biomedical Engineering, 30(10), 1273-1280. https://doi.org/10.1114/1.1528614

Marszalek, P E, Lu, H., Li, H. B., Carrion-Vazquez, M., Oberhauser, A. F., Schulten, K., \& Fernandez, J. M. (1999). Mechanical unfolding intermediates in titin modules. Nature, 402(6757), 100-103.

Marszalek, Piotr E, Li, H., \& Fernandez, J. M. (2001). Fingerprinting polysaccharides with singlemolecule atomic force microscopy. Nat Biotech, 19(3), 258-262.

Mennicke, U., \& Salditt, T. (2002). Preparation of solid-supported lipid bilayers by spin-coating. Langmuir, 18(21), 8172-8177. https://doi.org/10.1021/la025863f

Mierzwa, B. E., Chiaruttini, N., Redondo-Morata, L., von Filseck, J. M., Koenig, J., Larios, J., Poser, I., Mueller-Reichert, T., Scheuring, S., Roux, A., \& Gerlich, D. W. (2017). Dynamic subunit turnover in ESCRT-III assemblies is regulated by Vps4 to mediate membrane remodelling during cytokinesis. Nature Cell Biology, 19(7), 787-+. https://doi.org/10.1038/ncb3559

Mills, T. T., Huang, J., Feigenson, G. W., \& Nagle, J. F. (2009). Effects of cholesterol and unsaturated DOPC lipid on chain packing of saturated gel-phase DPPC bilayers. General Physiology and Biophysics, 28(2), 126-139.

Mingeot-Leclercq, M. P., Deleu, M., Brasseur, R., \& Dufrene, Y. F. (2008). Atomic force microscopy of supported lipid bilayers. Nature Protocols, 3(10), 1654-1659. https://doi.org/10.1038/nprot.2008.149

Miyoshi, T., Lönnfors, M., Peter Slotte, J., \& Kato, S. (2014). A detailed analysis of partial molecular volumes in DPPC/cholesterol binary bilayers. Biochimica Et Biophysica Acta, 1838(12), 3069-3077. https://doi.org/10.1016/j.bbamem.2014.07.004 
Morandat, S., Azouzi, S., Beauvais, E., Mastouri, A., \& El Kirat, K. (2013). Atomic force microscopy of model lipid membranes. Analytical and Bioanalytical Chemistry, 405(5), 1445-1461. https://doi.org/10.1007/s00216-012-6383-y

Mouritsen, O. G., \& Bloom, M. (1984). MATTRESS MODEL OF LIPID-PROTEIN INTERACTIONS IN MEMBRANES. Biophysical Journal, 46(2), 141-153.

Nagle, J. F., \& Tristram-Nagle, S. (2000). Structure of lipid bilayers. Biochimica Et Biophysica Acta-Reviews on Biomembranes, 1469(3), 159-195. https://doi.org/10.1016/s0304$4157(00) 00016-2$

Nawaz, S., Sánchez, P., Schmitt, S., Snaidero, N., Mitkovski, M., Velte, C., Brückner, B. R., Alexopoulos, I., Czopka, T., Jung, S. Y., Rhee, J. S., Janshoff, A., Witke, W., Schaap, I. A. T., Lyons, D. A., \& Simons, M. (2015). Actin Filament Turnover Drives Leading Edge Growth during Myelin Sheath Formation in the Central Nervous System. Developmental Cell, 34(2), 139-151. https://doi.org/10.1016/j.devcel.2015.05.013

Neupane, S., De Smet, Y., Renner, F. U., \& Losada-Pérez, P. (2018). Quartz Crystal Microbalance With Dissipation Monitoring: A Versatile Tool to Monitor Phase Transitions in Biomimetic Membranes. Frontiers in Materials, 5. https://doi.org/10.3389/fmats.2018.00046

Noy, A., Vezenov, D. V, \& Lieber, C. M. (1997). CHEMICAL FORCE MICROSCOPY. Annual Review of Materials Science, 27(1), 381-421. https://doi.org/10.1146/annurev.matsci.27.1.381

Olsson, A. L. J., Quevedo, I. R., He, D., Basnet, M., \& Tufenkji, N. (2013). Using the Quartz Crystal Microbalance with Dissipation Monitoring to Evaluate the Size of Nanoparticles Deposited on Surfaces. ACS Nano, 7(9), 7833-7843. https://doi.org/10.1021/nn402758w 
Pan, J., Mills, T. T., Tristram-Nagle, S., \& Nagle, J. F. (2008). Cholesterol perturbs lipid bilayers nonuniversally. Physical Review Letters, 100(19), 198103. https://doi.org/10.1103/PhysRevLett.100.198103

Parot, P., Dufrêne, Y. F., Hinterdorfer, P., Le Grimellec, C., Navajas, D., Pellequer, J. L., \& Scheuring, S. (2007). Past, present and future of atomic force microscopy in life sciences and medicine. J Mol Recognit, 20(6), 418-431. https://doi.org/10.1002/jmr.857

Peschel, A., Langhoff, A., Uhl, E., Dathathreyan, A., Haindl, S., Johannsmann, D., \& Reviakine, I. (2016). Lipid phase behavior studied with a quartz crystal microbalance: A technique for biophysical studies with applications in screening. The Journal of Chemical Physics, 145(20), 204904. https://doi.org/10.1063/1.4968215

Picas, L., Montero, M. T., Morros, A., Cabanas, M. E., Seantier, B., Milhiet, P.-E., \& HernandezBorrell, J. (2009). Calcium-Induced Formation of Subdomains in Phosphatidylethanolamine-Phosphatidylglycerol Bilayers: A Combined DSC, (31)P NMR, and AFM Study. Journal of Physical Chemistry B, 113(14), 4648-4655. https://doi.org/10.1021/jp8102468

Picas, L., Rico, F., \& Scheuring, S. (2012). Direct Measurement of the Mechanical Properties of Lipid Phases in Supported Bilayers. Biophysical Journal, 102(1), L1-L3. https://doi.org/10.1016/j.bpj.2011.11.4001

Piggot, T. J., Allison, J. R., Sessions, R. B., \& Essex, J. W. (2017). On the Calculation of Acyl Chain Order Parameters from Lipid Simulations. Journal of Chemical Theory and Computation, 13(11), 5683-5696. https://doi.org/10.1021/acs.jctc.7b00643

Pinkwart, K., Schneider, F., Lukoseviciute, M., Sauka-Spengler, T., Lyman, E., Eggeling, C., \& Sezgin, E. (2019). Nanoscale dynamics of cholesterol in the cell membrane. Journal of Biological Chemistry, 294(34), 12599-12609. https://doi.org/10.1074/jbc.RA119.009683 
Pramanik, S. K., Seneca, S., Ethirajan, A., Neupane, S., Renner, F. U., \& Losada-Pérez, P. (2016). Ionic strength dependent vesicle adsorption and phase behavior of anionic phospholipids on a gold substrate. Biointerphases, 11(1), 019006. https://doi.org/10.1116/1.4939596

Radmacher, M. (1997). Measuring the elastic properties of biological samples with the AFM. IEEE Engineering in Medicine and Biology Magazine, 16(2), 47-57. https://doi.org/10.1109/51.582176

Rascol, E., Devoisselle, J.-M., \& Chopineau, J. (2016). The relevance of membrane models to understand nanoparticles-cell membrane interactions. Nanoscale, 8(9), 4780-4798. https://doi.org/10.1039/C5NR07954C

Rawicz, W., Olbrich, K. C., McIntosh, T., Needham, D., \& Evans, E. (2000). Effect of chain length and unsaturation on elasticity of lipid bilayers. Biophysical Journal, 79(1), 328-339.

Redondo-Morata, L, Giannotti, M. I., \& Sanz, F. (2012a). AFM-Based Force-Clamp Monitors Lipid Bilayer Failure Kinetics. Langmuir, 28(15), 6403-6410. https://doi.org/10.1021/la3005147

Redondo-Morata, L, Giannotti, M. I., \& Sanz, F. (2012b). Influence of Cholesterol on the Phase Transition of Lipid Bilayers: A Temperature-Controlled Force Spectroscopy Study. Langmuir, 28(35), 12851-12860. https://doi.org/10.1021/la302620t

Redondo-Morata, L, Giannotti, M. I., \& Sanz, F. (2012c). Influence of Cholesterol on the Phase Transition of Lipid Bilayers: A Temperature-Controlled Force Spectroscopy Study. Langmuir, 28(35), 12851-12860. https://doi.org/10.1021/la302620t

Redondo-Morata, L., Giannotti, M. I., \& Sanz, F. (2012). Stability of Lipid Bilayers as Model Membranes: Atomic Force Microscopy and Spectroscopy Approach. In Atomic Force Microscopy in Liquid: Biological Applications. https://doi.org/10.1002/9783527649808.ch10 
Redondo-Morata, L, Giannotti, M. I., \& Sanz, F. (2014). Structural impact of cations on lipid bilayer models: Nanomechanical properties by AFM-force spectroscopy. Molecular Membrane Biology, 31(1), 17-28. https://doi.org/10.3109/09687688.2013.868940

Redondo-Morata, L, Sanford, R. L., Andersen, O. S., \& Scheuring, S. (2016). Effect of Statins on the Nanomechanical Properties of Supported Lipid Bilayers. Biophysical Journal, 111(2), 363-372. https://doi.org/10.1016/j.bpj.2016.06.016

Redondo-Morata, Lorena, Oncins, G., \& Sanz, F. (2012). Force Spectroscopy Reveals the Effect of Different lons in the Nanomechanical Behavior of Phospholipid Model Membranes: The Case of Potassium Cation. Biophysical Journal, 102(1), 66-74. https://doi.org/10.1016/j.bpj.2011.10.051

Reimhult, E., Hook, F., \& Kasemo, B. (2003). Intact vesicle adsorption and supported biomembrane formation from vesicles in solution: Influence of surface chemistry, vesicle size, temperature, and osmotic pressure. Langmuir, 19(5), 1681-1691. https://doi.org/10.1021/la0263920

Relat-Goberna, J., Beedle Amy, E. M., \& Garcia-Manyes, S. (2017). The Nanomechanics of Lipid Multibilayer Stacks Exhibits Complex Dynamics. Small, 13(24), 1700147. https://doi.org/10.1002/smll.201700147

Reviakine, I., Gallego, M., Johannsmann, D., \& Tellechea, E. (2012). Adsorbed liposome deformation studied with quartz crystal microbalance. The Journal of Chemical Physics, 136(8), 084702. https://doi.org/10.1063/1.3687351

Reviakine, I., Johannsmann, D., \& Richter, R. P. (2011). Hearing What You Cannot See and Visualizing What You Hear: Interpreting Quartz Crystal Microbalance Data from Solvated Interfaces. Analytical Chemistry, 83(23), 8838-8848. https://doi.org/10.1021/ac201778h 
1602

1603

1604

1605

1606

1607

1608

1609

1610

1611

1612

1613

1614

1615

1616

1617

1618

1619

1620

1621

1622

1623

1624

1625

1626

Richter, R., Mukhopadhyay, A., \& Brisson, A. (2003). Pathways of Lipid Vesicle Deposition on Solid Surfaces: A Combined QCM-D and AFM Study. Biophysical Journal, 85(5), 30353047.

Richter, R P. (2006). Formation of solid-supported lipid bilayers: An integrated view. Langmuir, 22(8), 3497-3505. https://doi.org/10.1021/la052687c

Richter, Ralf P, \& Brisson, A. (2003). Characterization of Lipid Bilayers and Protein Assemblies Supported on Rough Surfaces by Atomic Force Microscopy. Langmuir, 19(5), 16321640. https://doi.org/10.1021/la026427w

Richter, Ralf P, \& Brisson, A. R. (2005). Following the Formation of Supported Lipid Bilayers on Mica: A Study Combining AFM, QCM-D, and Ellipsometry. Biophysical Journal, 88(5), 3422-3433. https://doi.org/10.1529/biophysj.104.053728

Rief, M., Oesterhelt, F., Heymann, B., \& Gaub, H. E. (1997). Single Molecule Force Spectroscopy on Polysaccharides by Atomic Force Microscopy. Science, 275(5304), 1295 LP - 1297. https://doi.org/10.1126/science.275.5304.1295

Rog, T., Pasenkiewicz-Gierula, M., Vattulainen, I., \& Karttunen, M. (2009). Ordering effects of cholesterol and its analogues. Biochimica Et Biophysica Acta-Biomembranes, 1788(1), 97-121. https://doi.org/10.1016/j.bbamem.2008.08.022

Roux, A. (2013). The physics of membrane tubes: Soft templates for studying cellular membranes. Soft Matter, 9(29), 6726-6736.

Sauerbrey, G. (1959). Verwendung von Schwingquarzen zur Wägung dünner Schichten und zur Mikrowägung. Zeitschrift für Physik, 155(2), 206-222. https://doi.org/10.1007/BF01337937

Schmidtke, D. W., \& Diamond, S. L. (2000). Direct Observation of Membrane Tethers Formed during Neutrophil Attachment to Platelets or P-Selectin under Physiological Flow. The Journal of Cell Biology, 149(3), 719. 
Schneider, J., Dufrene, Y. F., Barger, W. R., \& Lee, G. U. (2000). Atomic force microscope image contrast mechanisms on supported lipid bilayers. Biophysical Journal, 79(2), 11071118.

Sebaaly, C., Greige-Gerges, H., \& Charcosset, C. (2019). Chapter 11-Lipid Membrane Models for Biomembrane Properties' Investigation (A. Basile \& C. B. T.-C. T. and F. D. on (Bio-) M. Charcosset, Eds.; pp. 311-340). Elsevier. https://doi.org/10.1016/B978-0-12813606-5.00011-7

Seeger, H. M., Di Cerbo, A., Alessandrini, A., \& Facci, P. (2010). Supported Lipid Bilayers on Mica and Silicon Oxide: Comparison of the Main Phase Transition Behavior. Journal of Physical Chemistry B, 114(27), 8926-8933. https://doi.org/10.1021/jp1026477

Sessa, G., \& Weissmann, G. (1968). Phospholipid spherules (liposomes) as a model for biological membranes. Journal of Lipid Research, 9(3), 310-318.

Shao, J.-Y., Ting-Beall, H. P., \& Hochmuth, R. M. (1998). Static and dynamic lengths of neutrophil microvilli. Proceedings of the National Academy of Sciences, 95(12), 6797.

Sheetz, M. P. (2001). Cell control by membrane-cytoskeleton adhesion. Nature Reviews Molecular Cell Biology, 2, 392.

Shinoda, K., Shinoda, W., \& Mikami, M. (2007). Molecular dynamics simulation of an archaeal lipid bilayer with sodium chloride. Physical Chemistry Chemical Physics, 9(5), 643-650. https://doi.org/10.1039/b611543h

Simons, K., \& Ikonen, E. (1997). Functional rafts in cell membranes. Nature, 387(6633), 569572. https://doi.org/10.1038/42408

Singer, S. J., \& Nicolson, G. L. (1972). FLUID MOSAIC MODEL OF STRUCTURE OF CELLMEMBRANES. Science, 175(4023), 720-. https://doi.org/10.1126/science.175.4023.720

Siontorou, C. G., Nikoleli, G.-P., Nikolelis, D. P., \& Karapetis, S. K. (2017). Artificial Lipid Membranes: Past, Present, and Future. Membranes, 7(3), 38. https://doi.org/10.3390/membranes7030038 
Steinkühler, J., Sezgin, E., Urbančič, I., Eggeling, C., \& Dimova, R. (2019). Mechanical properties of plasma membrane vesicles correlate with lipid order, viscosity and cell density. Communications Biology, 2(1), 1-8. https://doi.org/10.1038/s42003-019-0583-3

Strulson, M. K., \& Maurer, J. A. (2011). Microcontact printing for creation of patterned lipid bilayers on tetraethylene glycol self-assembled monolayers. Langmuir : The ACS Journal of Surfaces and Colloids, 27(19), 12052-12057. https://doi.org/10.1021/la201839w

Sullan, R M A, Li, J. K., Hao, C. C., Walker, G. C., \& Zou, S. (2010). Cholesterol-Dependent Nanomechanical Stability of Phase-Segregated Multicomponent Lipid Bilayers. Biophysical Journal, 99(2), 507-516. https://doi.org/10.1016/j.bpj.2010.04.044

Sullan, Ruby May A, Li, J. K., \& Zou, S. (2009a). Direct Correlation of Structures and Nanomechanical Properties of Multicomponent Lipid Bilayers. Langmuir, 25(13), 74717477. https://doi.org/10.1021/la900395w

Sullan, Ruby May A, Li, J. K., \& Zou, S. (2009b). Quantification of the Nanomechanical Stability of Ceramide-Enriched Domains. Langmuir, 25(22), 12874-12877. https://doi.org/10.1021/la903442s

Sun, M., Graham, J. S., Hegedüs, B., Marga, F., Zhang, Y., Forgacs, G., \& Grandbois, M. (2005). Multiple Membrane Tethers Probed by Atomic Force Microscopy. Biophysical Journal, 89(6), 4320-4329.

Tabaei, S. R., Gillissen, J. J. J., Vafaei, S., Groves, J. T., \& Cho, N.-J. (2016). Size-dependent, stochastic nature of lipid exchange between nano-vesicles and model membranes. Nanoscale, 8(27), 13513-13520. https://doi.org/10.1039/C6NR03817D

Tellechea, E., Johannsmann, D., Steinmetz, N. F., Richter, R. P., \& Reviakine, I. (2009). ModelIndependent Analysis of QCM Data on Colloidal Particle Adsorption. Langmuir, 25(9), 5177-5184. https://doi.org/10.1021/la803912p 
Tero, R. (2012). Substrate Effects on the Formation Process, Structure and Physicochemical Properties of Supported Lipid Bilayers. Materials, 5(12), 2658-2680. https://doi.org/10.3390/ma5122658

Van Lehn, R. C., Ricci, M., Silva, P. H. J., Andreozzi, P., Reguera, J., Voïtchovsky, K., Stellacci, F., \& Alexander-Katz, A. (2014). Lipid tail protrusions mediate the insertion of nanoparticles into model cell membranes. Nature Communications, 5(1), 4482. https://doi.org/10.1038/ncomms5482

van Meer, G., \& de Kroon, A. I. P. M. (2011). Lipid map of the mammalian cell. Journal of Cell Science, 124(1), 5.

van Meer, G., Voelker, D. R., \& Feigenson, G. W. (2008). Membrane lipids: Where they are and how they behave. Nature Reviews Molecular Cell Biology, 9(2), 112-124. https://doi.org/10.1038/nrm2330

Veatch, S. L., \& Keller, S. L. (2003). Separation of Liquid Phases in Giant Vesicles of Ternary Mixtures of Phospholipids and Cholesterol. Biophysical Journal, 85(5), 3074-3083.

Vereb, G., Szöllősi, J., Matkó, J., Nagy, P., Farkas, T., Vígh, L., Mátyus, L., Waldmann, T. A., \& Damjanovich, S. (2003). Dynamic, yet structured: The cell membrane three decades after the Singer-Nicolson model. Proceedings of the National Academy of Sciences, 100(14), 8053 LP - 8058. https://doi.org/10.1073/pnas.1332550100

Vist, M. R., \& Davis, J. H. (1990). Phase equilibria of cholesterol/dipalmitoylphosphatidylcholine mixtures: Deuterium nuclear magnetic resonance and differential scanning calorimetry. Biochemistry, 29(2), 451-464. https://doi.org/10.1021/bi00454a021

Vogel, V. (2006). MECHANOTRANSDUCTION INVOLVING MULTIMODULAR PROTEINS: Converting Force into Biochemical Signals. Annual Review of Biophysics and Biomolecular Structure, 35(1), 459-488. https://doi.org/10.1146/annurev.biophys.35.040405.102013 
Voinova, M. V., Rodahl, M., Jonson, M., \& Kasemo, B. (1999). Viscoelastic Acoustic Response of Layered Polymer Films at Fluid-Solid Interfaces: Continuum Mechanics Approach. Physica Scripta, 59(5), 391. https://doi.org/10.1238/Physica.Regular.059a00391

Wallace, E. J., Hooper, N. M., \& Olmsted, P. D. (2006). Effect of hydrophobic mismatch on phase behavior of lipid membranes. Biophysical Journal, 90(11), 4104-4118. https://doi.org/10.1529/biophysj.105.062778

Wargenau, A., \& Tufenkji, N. (2014). Direct Detection of the Gel-Fluid Phase Transition of a Single Supported Phospholipid Bilayer Using Quartz Crystal Microbalance with Dissipation Monitoring. Analytical Chemistry, 86(16), 8017-8020. https://doi.org/10.1021/ac5019183

Winkler, P. M., Campelo, F., Giannotti, M. I., \& García-Parajo, M. F. (2020). Planar plasmonic antenna arrays resolve transient nanoscopic heterogeneities in biological membranes. Proc.SPIE, 11246. https://doi.org/10.1117/12.2543726

Winkler, P. M., Regmi, R., Flauraud, V., Brugger, J., Rigneault, H., Wenger, J., \& García-Parajo, M. F. (2017). Transient Nanoscopic Phase Separation in Biological Lipid Membranes Resolved by Planar Plasmonic Antennas. ACS Nano, 11(7), 7241-7250. https://doi.org/10.1021/acsnano.7b03177

Yeagle, P. L. (1989). Lipid regulation of cell membrane structure and function. The FASEB Journal, 3(7), 1833-1842. https://doi.org/10.1096/fasebj.3.7.2469614

Zou, S., \& Johnston, L. J. (2010). Ceramide-enriched microdomains in planar membranes. Current Opinion in Colloid \& Interface Science, 15(6), 489-498. https://doi.org/10.1016/j.cocis.2010.06.003 
L. Redondo-Morata, P. Losada-Pérez \& M. I. Giannotti

1729

- 64 - I "Lipid bilayers: phase behavior and nanomechanics" in Membrane Biomechanics, Volume 86. Ed. Levitan, I.; Trache, A.; 2020. ISBN: 9780128210215 , Elsevier 